\title{
Precision neutrino experiments vs the Littlest Seesaw
}

\author{
Peter Ballett, ${ }^{a}$ Stephen F. King, ${ }^{b}$ Silvia Pascoli, ${ }^{a}$ Nick W. Prouse ${ }^{b, c}$ \\ and TseChun Wang ${ }^{a}$ \\ ${ }^{a}$ Institute for Particle Physics Phenomenology, \\ Department of Physics, Durham University, \\ South Road, Durham DH1 3LE, United Kingdom \\ ${ }^{b}$ School of Physics and Astronomy, University of Southampton, \\ SO17 1BJ Southampton, United Kingdom \\ ${ }^{c}$ Particle Physics Research Centre, School of Physics and Astronomy, \\ Queen Mary University of London, \\ Mile End Road, London E1 4NS, United Kingdom \\ E-mail: peter.ballett@durham.ac.uk, king@soton.ac.uk, \\ silvia.pascoli@durham.ac.uk, n.prouse@soton.ac.uk, \\ tse-chun.wang@durham.ac.uk
}

ABSTRACT: We study to what extent upcoming precision neutrino oscillation experiments will be able to exclude one of the most predictive models of neutrino mass and mixing: the Littlest Seesaw. We show that this model provides a good fit to current data, predicting eight observables from two input parameters, and provide new assessments of its predictions and their correlations. We then assess the ability to exclude this model using simulations of upcoming neutrino oscillation experiments including the medium-distance reactor experiments JUNO and RENO-50 and the long-baseline accelerator experiments DUNE and T2HK. We find that an accurate determination of the currently least well measured parameters, namely the atmospheric and solar angles and the $\mathrm{CP}$ phase $\delta$, provide crucial independent tests of the model. For $\theta_{13}$ and the two mass-squared differences, however, the model's exclusion requires a combination of measurements coming from a varied experimental programme. Our results show that the synergy and complementarity of future experiments will play a vital role in efficiently discriminating between predictive models of neutrino flavour, and hence, towards advancing our understanding of neutrino oscillations in the context of the flavour puzzle of the Standard Model.

Keywords: Neutrino Physics, Solar and Atmospheric Neutrinos

ArXiv EPrint: 1612.01999 


\section{Contents}

1 Introduction 1

2 Littlest Seesaw models of neutrinos 3

2.1 Sum rules of LS 5

3 Probing LS with existing data $\quad 6$

3.1 Predictions of oscillation parameters with fixed $\eta= \pm 2 \pi / 3 \quad 6$

3.2 Predictions of oscillation parameters with $\eta$ as a free parameter 8

3.3 Fitting LS models to global fit data 9

4 Sensitivity of future experiments $\quad 13$

$\begin{array}{lll}4.1 & \text { Future neutrino oscillation experiments } & 13\end{array}$

$\begin{array}{ll}4.2 \text { Statistical method } & 16\end{array}$

$\begin{array}{lll}4.3 & \text { Results } & 17\end{array}$

$\begin{array}{llr}5 & \text { Conclusion } & 20\end{array}$

A Exact expressions for LS sum rules $\quad 24$

\section{Introduction}

The framework of neutrino masses and mixing for explaining neutrino oscillations - the first direct experimental evidence for physics beyond the Standard Model — is now firmly established [1]. All three mixing angles together with the size of the two mass-squared differences have been measured, with experimental efforts now focused on determining the final few unknowns: the ordering and scale of the neutrino masses; the value of the Dirac phase $\delta$; and a precision measurement of the angle $\theta_{23}$ including, if non-maximal, its octant. Although there is some as yet inconclusive evidence for $\delta$ in the third or fourth quadrant, as well as for normal ordering (NO) and non-maximal atmospheric mixing, we rely on the next generation of oscillation experiments to set these issues to rest.

On the theoretical side, however, the origin of neutrino masses and mixing remains unknown with many possible models considered viable (for reviews see e.g. [2-6]). A large proportion of these models are based on the classic seesaw mechanism, involving heavy right-handed Majorana neutrinos [7-12], providing both a mechanism for generating the neutrino masses and a natural explanation for their smallness. However, in order to make predictions that can be probed experimentally, seesaw models require additional assumptions or constraints [13].

To accommodate the three distinct light neutrino masses which drive the oscillation phenomenon, the seesaw mechanism requires at least two right-handed neutrinos [14]. In 
order to reduce the number of free parameters still further to the smallest number possible, and hence increase predictivity, various approaches to the two right-handed neutrino seesaw model have been suggested, ${ }^{1}$ such as postulating one [15] or two [16] texture zeroes in the Dirac mass matrix in the flavour basis (i.e. the basis of diagonal charged lepton and right-handed neutrino masses). However, such two texture zero models are now phenomenologically excluded [17] for the case of a normal neutrino mass hierarchy. The minimal two right-handed neutrino model with normal hierarchy which can accommodate the known data of neutrino mixing involves a Dirac mass matrix with one texture zero and a characteristic form known as the Littlest Seesaw model [18]. The Littlest Seesaw model may be embedded in unified models of quarks and leptons in [19, 20]. It leads to successful leptogenesis where the sign of baryon asymmetry is determined by the ordering of the heavy right-handed neutrinos, and the only seesaw phase $\eta$ is identified as the leptogenesis phase, linking violation of charge parity symmetry $(\mathrm{CP})$ in the laboratory with that in the early universe [21].

The Littlest Seesaw model can be understood as an example of sequential dominance (SD) $[22,23]$ in which one right-handed neutrino provides the dominant contribution to the atmospheric neutrino mass, ${ }^{2}$ leading to approximately maximal atmospheric mixing, while the other right-handed neutrino gives the solar neutrino mass and controls the solar and reactor mixing as well as the magnitude of $\mathrm{CP}$ violating effects via $\delta$. SD generally leads to normal ordering and a reactor angle which is bounded by $\theta_{13} \lesssim m_{2} / m_{3}$ [15], proposed a decade before the reactor angle was measured [1]. Precise predictions for the reactor (and solar) angles result from applying further constraints to the Dirac mass matrix, an approach known as constrained sequential dominance (CSD) [24]. For example, keeping the first column of the Dirac mass matrix proportional to $(0,1,1)^{T}$, a class of $\operatorname{CSD}(n)$ models has emerged [18, 24-29] corresponding to the second column proportional to $(1, n,(n-2))^{T}$, with a reactor angle approximately given by [30] $\theta_{13} \sim(n-1) \frac{\sqrt{2}}{3} \frac{m_{2}}{m_{3}}$. The Littlest Seesaw model corresponds to $n=3$ with a fixed seesaw phase $\eta=2 \pi / 3$.

It was recently realised that the alternative form of the Littlest Seesaw model with second column $(1,1,3)^{T}$ and seesaw phase $\eta=-2 \pi / 3$ (also proposed in [18]) may be enforced by an $S_{4} \times \mathrm{U}(1)$ symmetry, putting this version of the Littlest Seesaw model on a firm theoretical foundation [31] in which the required vacuum alignment emerges from symmetry as a semi-direct model [32]. In general the Littlest Seesaw model is an example of trimaximal $\mathrm{TM}_{1}$ mixing [33-39], in which the first column of the tri-bimaximal mixing matrix [40] is preserved, similar to the semi-direct model of trimaximal $\mathrm{TM}_{1}$ mixing that was developed in [41]. To fix the seesaw phase, one imposes a CP symmetry in the original theory which is spontaneously broken, where, unlike [42, 43], there is no residual CP symmetry in either the charged lepton or neutrino sectors, but instead the phase $\eta$ in the neutrino mass matrix is fixed to be one of the cube roots of unity due to a $Z_{3}$ family symmetry, using the mechanism proposed in [44].

\footnotetext{
${ }^{1}$ In seesaw models with two right-handed neutrinos, including those discussed in this paper, a hierarchical spectrum of left-handed neutrino masses is obtained where the lightest left-handed neutrino is massless.

${ }^{2}$ With the lightest neutrino massless, $m_{1}=0$, we refer to the two non-zero masses as the solar neutrino mass and the atmospheric neutrino mass, corresponding to the square roots of the experimentally measured solar and atmospheric neutrino mass splittings $m_{2}=\sqrt{\Delta m_{21}^{2}}$ and $m_{3}=\sqrt{\Delta m_{31}^{2}}$ respectively.
} 
As explained in more detail later on, the Littlest Seesaw model predicts all neutrino masses and mixing parameters in terms of two or three parameters, and it has been shown that the model is in agreement with all existing data, for a suitable range of its internal parameters [29]. The model makes some key predictions about the neutrino mass spectrum, that the lightest neutrino is massless $m_{1}=0$ and that normal ordering ${ }^{3}$ obtains $\Delta m_{31}^{2}>0$, which offer a means to exclude it via the observation of neutrinoless double beta decay, ${ }^{4}$ the measurement of the beta-decay end-point, or from cosmological measurements, as well as any measurement of NO from neutrino oscillation searches. However, the model also provides a rich set of predictions and correlations for the mixing angles and phases. In this paper, we assume Normal Hierarchy $\left(m_{1}=0\right.$ and NO), and study how the future longand medium-baseline oscillation programme will be able to test this model through the precision measurement of the oscillation parameters.

The layout of the paper is as follows: in section 2 we define the Littlest Seesaw models discussed above, and express some of the predictions in terms of exact sum rules of the neutrino oscillation parameters. In section 3 the Littlest Seesaw models are confronted with existing oscillation data and we show the precise predictions made once this data is taken into account. Section 4 then covers how the predictions of the models could be probed at future experimental facilities, showing the sensitivities of experiments to exclude the models and the combined measurements required to do so. We end with some concluding remarks in section 5 .

\section{Littlest Seesaw models of neutrinos}

Sequential dominance models of neutrinos arise from the proposal that, via the type-I seesaw mechanism, a dominant heavy right-handed $(\mathrm{RH})$ neutrino is mainly responsible for the atmospheric neutrino mass, a heavier subdominant RH neutrino for the solar neutrino mass, and a possible third largely decoupled $\mathrm{RH}$ neutrino for the lightest neutrino mass $[22,23]$. This leads to the prediction of normal neutrino mass ordering and, in the minimal case containing just the dominant and subdominant right-handed neutrinos, the lightest neutrino must be massless. Constrained sequential dominance (CSD) constrains these models further through the introduction of flavour symmetry, with the indirect approach used to fix the mass matrix from vacuum alignments of flavon fields [24]. A family of such models, parameterized by $n$, either integer or real using the flavour symmetry groups $S_{4}$ or $A_{4}$ respectively, predicts the $\operatorname{CSD}(n)$ mass matrix for left-handed neutrinos $[18,30]$. After integrating out the heavy neutrinos, the resulting left-handed light effective Majorana neutrino mass matrix ${ }^{5}$ in the charged-lepton flavour basis is given by

$$
m^{\nu}=m_{a}\left(\begin{array}{lll}
0 & 0 & 0 \\
0 & 1 & 1 \\
0 & 1 & 1
\end{array}\right)+m_{b} e^{i \eta}\left(\begin{array}{ccc}
1 & n & (n-2) \\
n & n^{2} & n(n-2) \\
(n-2) & n(n-2) & (n-2)^{2}
\end{array}\right),
$$

\footnotetext{
${ }^{3}$ The prediction of NO is a general consequence of Sequential Dominance upon which the model is built $[22,23]$.

${ }^{4}$ The masslessness of the lightest neutrino leads to a neutrinoless double beta decay rate far smaller than could foreseeably be observed [29].

${ }^{5}$ We follow the Majorana mass Lagrangian convention $-\frac{1}{2} \overline{\nu_{L}} m^{\nu} \nu_{L}^{c}$.
} 
where in addition to $n$ there are three free real parameters: two parameters with the dimension of mass $m_{a}$ and $m_{b}$ which are proportional to the reciprocal of the masses of the dominant and subdominant right-handed neutrinos, and a relative phase $\eta$. A second version of this model has also been proposed, based on an $S_{4} \times \mathrm{U}(1)$ symmetry, where the second and third rows and columns of the mass matrix are swapped [31]. In this paper, we discuss both these versions for the case where $n=3$ since it has been shown that $n=4$ is disfavoured and other values of $n$ are already excluded [29,31]. These models are also known as Littlest Seesaw (LS) models since they provide physically viable seesaw models with the fewest number of free parameters. We use the two versions of the model denoted as LSA and LSB;

$$
\begin{aligned}
& m_{\mathrm{LSA}}^{\nu}=m_{a}\left(\begin{array}{lll}
0 & 0 & 0 \\
0 & 1 & 1 \\
0 & 1 & 1
\end{array}\right)+m_{b} e^{i \eta}\left(\begin{array}{lll}
1 & 3 & 1 \\
3 & 9 & 3 \\
1 & 3 & 1
\end{array}\right) \\
& m_{\mathrm{LSB}}^{\nu}=m_{a}\left(\begin{array}{lll}
0 & 0 & 0 \\
0 & 1 & 1 \\
0 & 1 & 1
\end{array}\right)+m_{b} e^{i \eta}\left(\begin{array}{lll}
1 & 1 & 3 \\
1 & 1 & 3 \\
3 & 3 & 9
\end{array}\right)
\end{aligned}
$$

Although, in the most minimal set-up, the relative phase $\eta$ is a free parameter, it has been shown that in some models the presence of additional $Z_{3}$ symmetries can fix the phase $e^{i \eta}$ to a cube root of unity [42,43], with $\eta=2 \pi / 3$ the preferred value for LSA and $\eta=-2 \pi / 3$ for LSB as determined by current data [29]. This restriction gives the model greater predictivity by reducing the number of free parameters to two, and we will give these cases special attention while also showing some results for the case with $\eta$ left free.

Diagonalizing the mass matrices above leads to predictions for the neutrino masses as well as the angles and phases of the unitary PMNS matrix, $U_{\mathrm{PMNS}}$, which describes the mixing between the three left-handed neutrinos

$$
U_{\mathrm{PMNS}}^{T} m^{\nu} U_{\mathrm{PMNS}}=\left(\begin{array}{ccc}
m_{1} & 0 & 0 \\
0 & m_{2} & 0 \\
0 & 0 & m_{3}
\end{array}\right)
$$

where $U_{\text {PMNS }}$ is defined by

$$
U_{\mathrm{PMNS}}=\left(\begin{array}{ccc}
c_{12} c_{13} & s_{12} c_{13} & s_{13} e^{-i \delta} \\
-s_{12} c_{23}-c_{12} s_{13} s_{23} e^{i \delta} & c_{12} c_{23}-s_{12} s_{13} s_{23} e^{i \delta} & c_{13} s_{23} \\
s_{12} s_{23}-c_{12} s_{13} c_{23} e^{i \delta} & -c_{12} s_{23}-s_{12} s_{13} c_{23} e^{i \delta} & c_{13} c_{23}
\end{array}\right)\left(\begin{array}{ccc}
e^{i \frac{\beta_{1}}{2}} & 0 & 0 \\
0 & e^{i \frac{\beta_{2}}{2}} & 0 \\
0 & 0 & 1
\end{array}\right)
$$

with $s_{i j}=\sin \theta_{i j}$ and $c_{i j}=\cos \theta_{i j}$. All of the parameters in this decomposition are therefore predicted in terms of the 2 (or 3 ) real parameters in eqs. (2.2) and (2.3). Due to the minimal assumption of only two right-handed neutrinos, the lightest neutrino is massless $m_{1}=0$ and the mass-squared differences, which are the only combinations of masses accessible to neutrino oscillation experiments, are predicted to be $\Delta m_{21}^{2}=m_{2}^{2}$ and $\Delta m_{31}^{2}=m_{3}^{2}$. Of the remaining mixing parameters, $\theta_{12}, \theta_{13}, \theta_{23}$ and $\delta$, are also experimentally accessible via neutrino oscillation, while the Majorana phases $\beta_{1}$ and $\beta_{2}$ are not. 
As will be seen in more detail in the next section, due to their similar forms, LSA and LSB make similar predictions. However, the process of diagonalization reveals that the octant of $\theta_{23}$ is reversed, along with the sign of $\delta$, while all other parameters are unchanged. Changing the sign of $\eta$, however, also reverses the sign of $\delta$ with no other effect, and so with the sign of $\eta$ not fixed by the model the only physical difference between LSA and LSB is the octant of $\theta_{23}$.

\subsection{Sum rules of LS}

It has already been shown that, since the first column of the LS mixing matrix $U_{\text {PMNS }}$ is equal to that of the tri-bimaximal mixing matrix, LS (both LSA and LSB for all values of $\eta$ ) obeys the TM1 sum rules $[30,31]$

$$
\begin{aligned}
\tan \theta_{12} & =\frac{1}{\sqrt{2}} \sqrt{1-3 s_{13}^{2}}, \quad \sin \theta_{12}=\frac{1}{\sqrt{3}} \frac{\sqrt{1-3 s_{13}^{2}}}{c_{13}}, \quad \cos \theta_{12}=\sqrt{\frac{2}{3}} \frac{1}{c_{13}}, \\
\cos \delta & =-\frac{\cot 2 \theta_{23}\left(1-5 s_{13}^{2}\right)}{2 \sqrt{2} s_{13} \sqrt{1-3 s_{13}^{2}}},
\end{aligned}
$$

where $s_{i j}=\sin \theta_{i j}$ and $c_{i j}=\cos \theta_{i j}$, and the forms in eq. (2.6) are equivalent.

For LSA with $\eta=\frac{2 \pi}{3}$ or LSB with $\eta=-\frac{2 \pi}{3}$, there are several additional sum rules, which we have derived and present here for the first time. A set of these additional sum rules can be derived using the fact that the only two remaining input parameters $m_{a}$ and $m_{b}$ have dimensions of mass, so all the mixing angles and phases must depend only on the ratio $r \equiv \frac{m_{b}}{m_{a}}$. Exact expressions for the mixing angles and Dirac phase as a function of $r$ can be found in appendix A, along with new exact sum rules derived using these expressions. These results make clear the difference between predictions of LSA and LSB; while $\theta_{13}$ and $\theta_{12}$ remain unchanged, $\cos 2 \theta_{23}$ and $\cos \delta$ differ by a change of sign.

An exact expression for the Jarlskog invariant $J$ has previously been given as [30, 31]

$$
J=s_{12} c_{12} s_{13} c_{13}^{2} s_{23} c_{23} \sin \delta=\mp \frac{24 m_{a}^{3} m_{b}^{3}(n-1) \sin \eta}{m_{3}^{2} m_{2}^{2} \Delta m_{32}^{2}},
$$

with negative sign taken for LSA and positive for LSB. For both LSA with $\eta=\frac{2 \pi}{3}$, and LSB with $\eta=-\frac{2 \pi}{3}$ we find the new relation

$$
m_{2} m_{3}=6 m_{a} m_{b}
$$

Using this relation and inserting $n=3$ into eq. (2.8) leads to the new relation for the Jarlskog invariant $J$

$$
J=-\frac{\sqrt{\Delta m_{21}^{2} \Delta m_{31}^{2}}}{3 \sqrt{3} \Delta m_{32}^{2}}
$$

and hence the new sum rule,

$$
\sin \delta=-\frac{\sqrt{\Delta m_{21}^{2} \Delta m_{31}^{2}}}{3 \sqrt{3} \Delta m_{32}^{2} s_{12} c_{12} s_{13} c_{13}^{2} s_{23} c_{23}},
$$

which is valid for both LSA with $\eta=\frac{2 \pi}{3}$ and LSB with $\eta=-\frac{2 \pi}{3}$. 

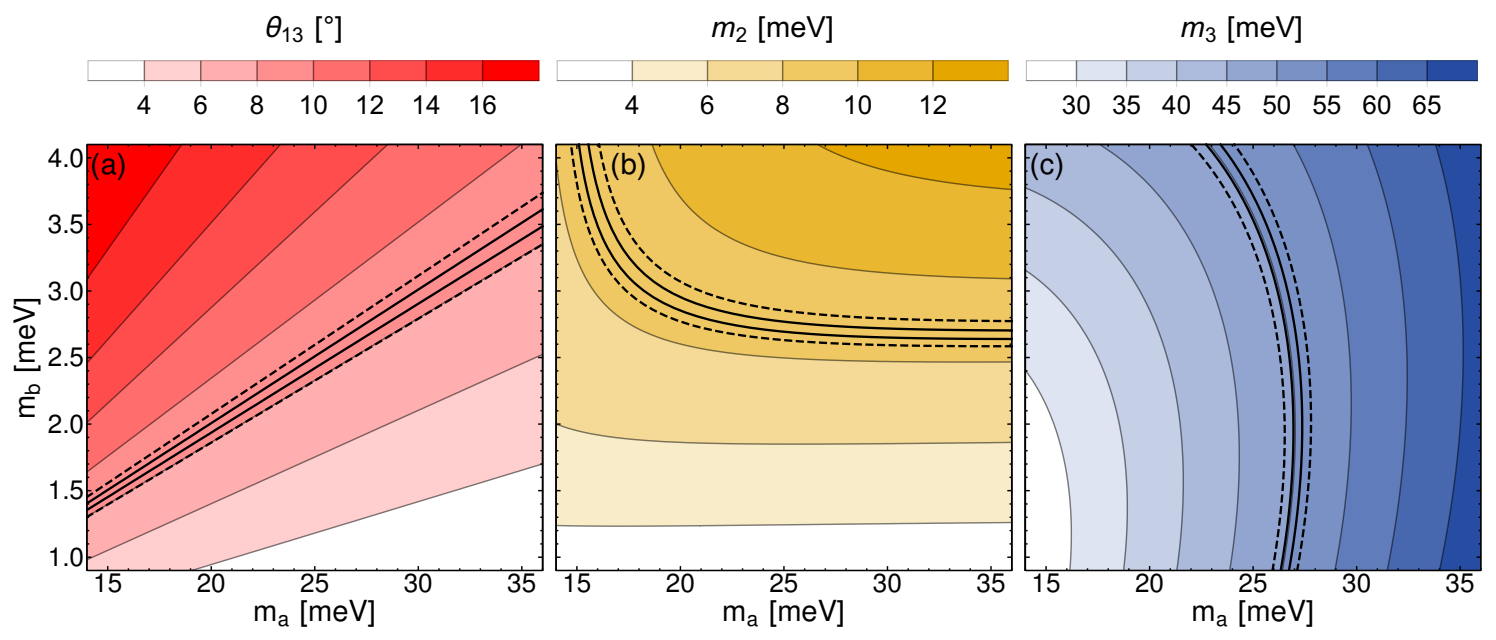

Figure 1. Predicted values from LSA with $\eta=\frac{2 \pi}{3}$ (or LSB with $\eta=-\frac{2 \pi}{3}$ ) of oscillation parameters depending on the input parameters $m_{a}$ and $m_{b}$. Regions corresponding to the experimentally determined $1 \sigma$ (solid lines) and $3 \sigma$ (dashed lines) ranges for each parameter are also shown.

\section{Probing LS with existing data}

Existing measurements of the neutrino mixing parameters have been shown to be in good agreement for $\operatorname{CSD}(n)$ for the $n=3$ case [29]. The best-fit value of $\eta$ is found to be close to $\pm \frac{2 \pi}{3}$, with the positive sign for LSA and the negative sign for LSB, which has been theoretically motivated as one of the cube roots of unity required due to an additional $Z_{3}$ symmetry as part of a larger GUT model [30]. In this section, we study both the case where $\eta$ is fixed by symmetry and the case where it is left as a free parameter of the theory.

\subsection{Predictions of oscillation parameters with fixed $\eta= \pm 2 \pi / 3$}

In the $n=3$ case of LSA with $\eta=\frac{2 \pi}{3}$ (or LSB with $\eta=-\frac{2 \pi}{3}$ ), all neutrino masses, mixing angles and phases are fully determined from the two remaining parameters $m_{a}$ and $m_{b}$ and the three most precisely measured of these parameters, $\theta_{13}, \Delta m_{31}^{2}$ and $\Delta m_{21}^{2}$, currently provide the strongest test of the LS model. We use exact expressions for all mixing angles and neutrino masses, derived in [30, 31], to study these predictions of the LSA and LSB models. Figure 1 shows how these parameters vary in the $m_{a}-m_{b}$ plane, along with the regions corresponding to the $1 \sigma$ and $3 \sigma$ ranges for these parameters from the NuFit 3.0 (2016) global fit [46], assuming normal mass ordering and a lightest neutrino mass of $m_{1}=0$. The SD proposal requires $m_{a}$ to be significantly larger than $m_{b}$ and for this portion of the parameter space the approximate proportionality relations of $m_{2} \sim m_{b}$ and $m_{3} \sim m_{a}$ can be seen, verifying the approximations previously derived in [30].

Even at $1 \sigma$ the three allowed regions coincide at a single point, as can be seen in figure 2, and so this benchmark point can be used to make predictions of the remaining angles $\theta_{12}$ and $\theta_{23}$ and the Dirac phase $\delta$. As described in section 2 these parameters, along with $\theta_{13}$, depend only on the ratio $r=m_{b} / m_{a}$; this dependence, given by the relations in eq. (A.1), is shown in figure 3, with the $1 \sigma$ and $3 \sigma$ NuFIT 3.0 ranges and reference point 


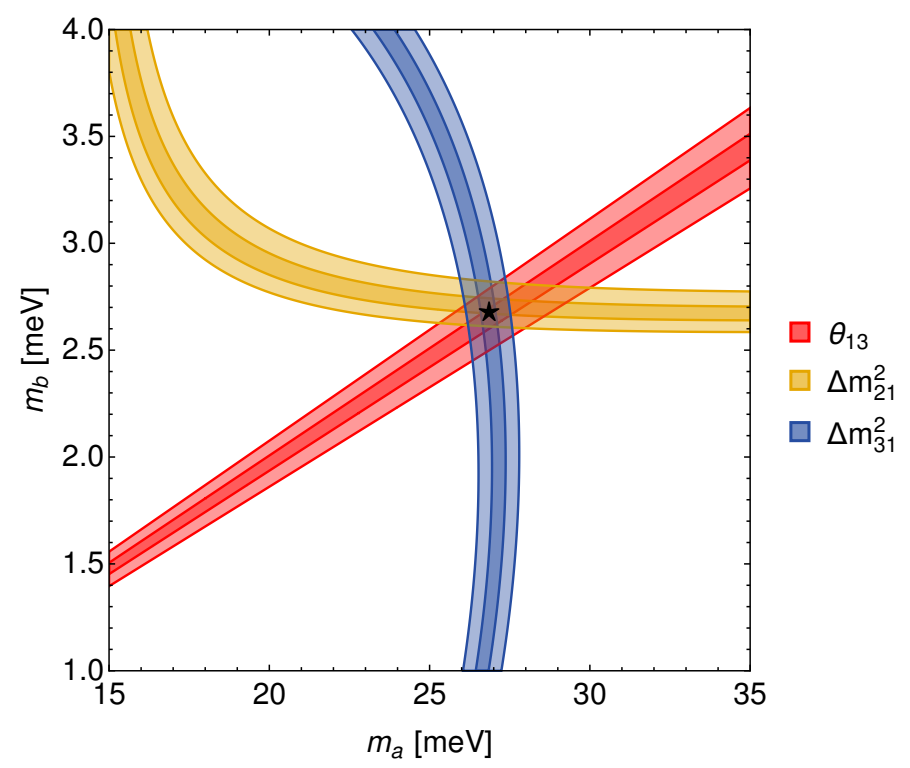

Figure 2. Regions in the $m_{a}-m_{b}$ plane with fixed $\eta=2 \pi / 3(\eta=-2 \pi / 3)$ for LSA (LSB) corresponding to the experimentally determined $1 \sigma$ and $3 \sigma$ ranges for $\theta_{13}, \Delta m_{21}^{2}$ and $\Delta m_{31}^{2}$.
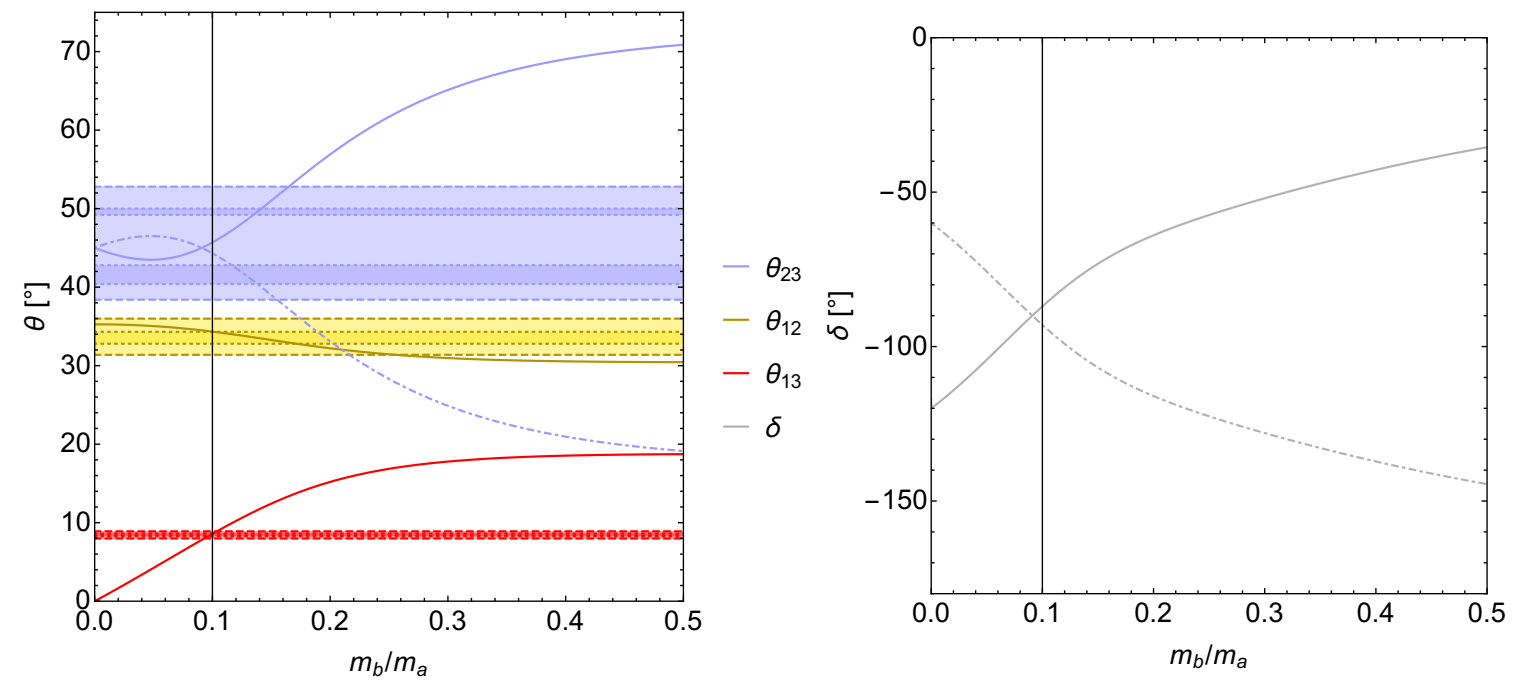

Figure 3. Predicted values from LS with fixed $\eta=2 \pi / 3$ for LSA (solid) and $\eta=-2 \pi / 3$ for LSB (dot-dashed) of the mixing angles and delta as a function of the ratio $m_{b} / m_{a}$. Note that only $\theta_{23}$ and $\delta$ differ between LSA and LSB. Horizontal bands show the experimentally determined $1 \sigma$ and $3 \sigma$ ranges for each parameter. A reference point giving a good prediction for all parameters is shown at $r=m_{b} / m_{a}=0.1$.

at $m_{b} / m_{a}=0.1$. For $\theta_{23}$ and $\delta$, the predictions of both LSA and LSB are shown. At this point it can be seen that while both $\theta_{13}$ and $\theta_{12}$ lie within their $1 \sigma$ ranges, $\theta_{23}$ lies just outside its $1 \sigma$ range, and a prediction on the value of the Dirac phase is made of $\delta \simeq-90^{\circ}$.

Combining these results for all parameters which have been experimentally measured, displayed together in figure 4 , it is seen that the prediction for $\theta_{12}$ lies just within current bounds. However, there is tension at the $1 \sigma$ level for $\theta_{23}$, due to the allowed regions of LS 


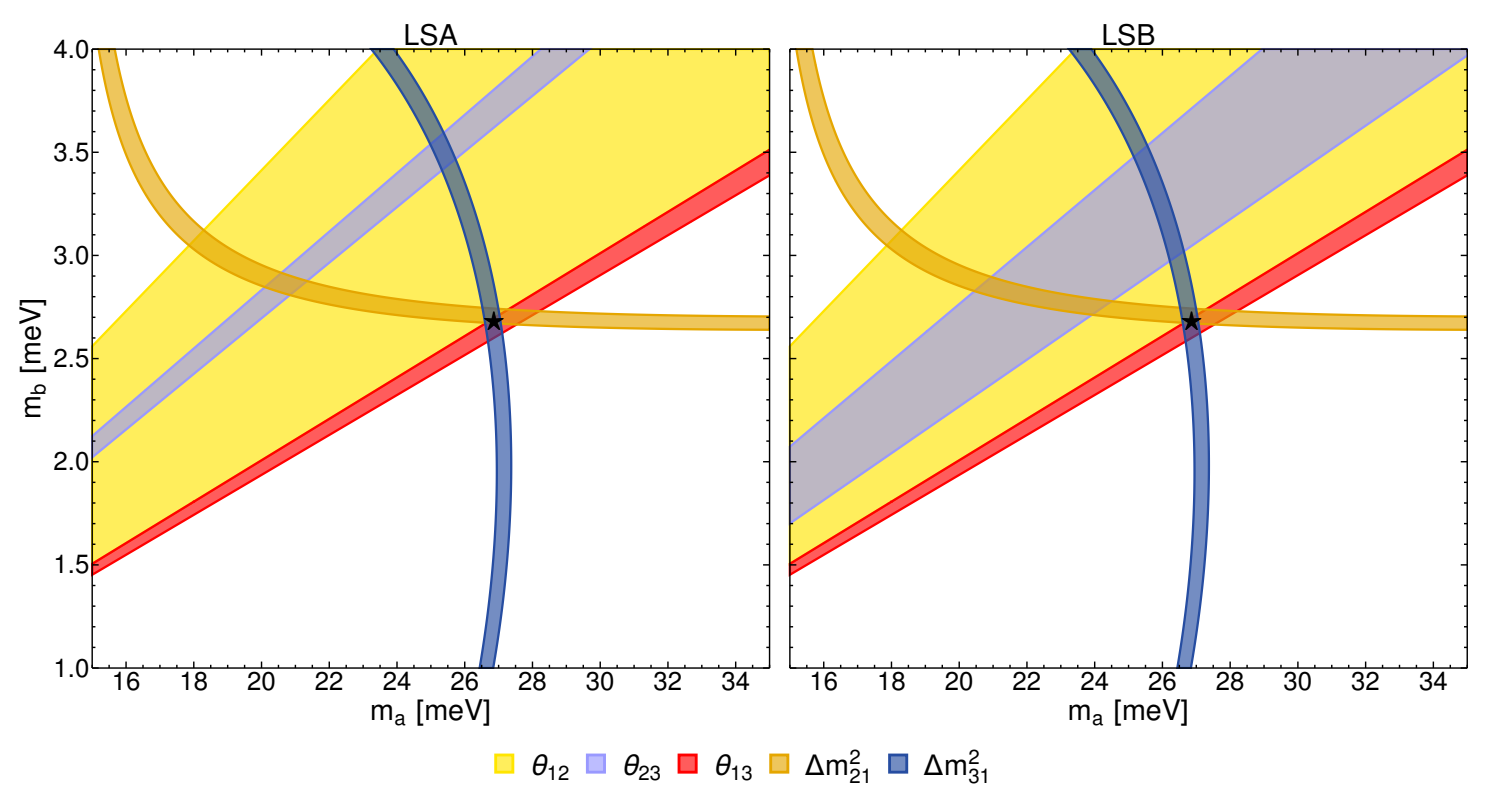

Figure 4. Regions in the $m_{a}-m_{b}$ plane with fixed $\eta=2 \pi / 3(\eta=-2 \pi / 3)$ for LSA (LSB) corresponding to the experimentally determined $1 \sigma$ ranges for solar and reactor mixing angles and mass-squared differences. The $\theta_{23}$ regions shown are in tension with other measurements, however, extending to $2 \sigma$ these regions become far larger, covering the entire parameter space shown in these plots.

parameter space requiring values close to maximal, while current data points towards larger deviations from the maximal value. The experimental measurements of $\theta_{23}$ do not yet give consistent indications of its value; while the latest results from $\mathrm{NO} \nu \mathrm{A}$ disfavour maximal mixing at $2.5 \sigma$ [48], results from T2K remain fully compatible with maximal $\theta_{23}$ [47]. As a result, while the combined fit for $\theta_{23}$ is in tension with the LS models at $1 \sigma$, the allowed range at $2 \sigma$ is far wider, crossing both octants and the maximal value of $45^{\circ}$, including the values preferred by the LS model. ${ }^{6}$

\subsection{Predictions of oscillation parameters with $\eta$ as a free parameter}

In the versions of the LS models with $\eta$ as an additional free parameter, the mixing angles and phases now depend on both the ratio $r=m_{b} / m_{a}$ and $\eta$. The masses $m_{3}$ and $m_{2}$ depend on all three input parameters; however, their ratio $m_{2} / m_{3}$ (and therefore the ratio $\left.\Delta m_{21}^{2} / \Delta m_{31}^{2}\right)$ will depend only on $r$ and $\eta$. As previously, the strongest contraints come from the very precise measurements of $\theta_{13}$ and the mass-squared differences $\Delta m_{21}^{2}$ and $\Delta m_{31}^{2}$. Figure 5 shows the regions corresponding to the $1 \sigma$ ranges for all the mixing angles, $\delta$ and $m_{2} / m_{3}$, where we see that all the five regions come close to overlapping around $\eta= \pm 2 \pi / 3$ for LSA and LSB, respectively. That two input parameters should give a good description of five observables, within their one sigma errors, is ostensibly a remarkable achievement, indeed perhaps better than might be expected on statistical grounds. However, due to the very tight constraints on $\eta$ from $\theta_{13}$ and $m_{2} / m_{3}$, we still find

\footnotetext{
${ }^{6}$ For a more detailed discussion of the current status of experimental measurements of $\theta_{23}$, see [46].
} 


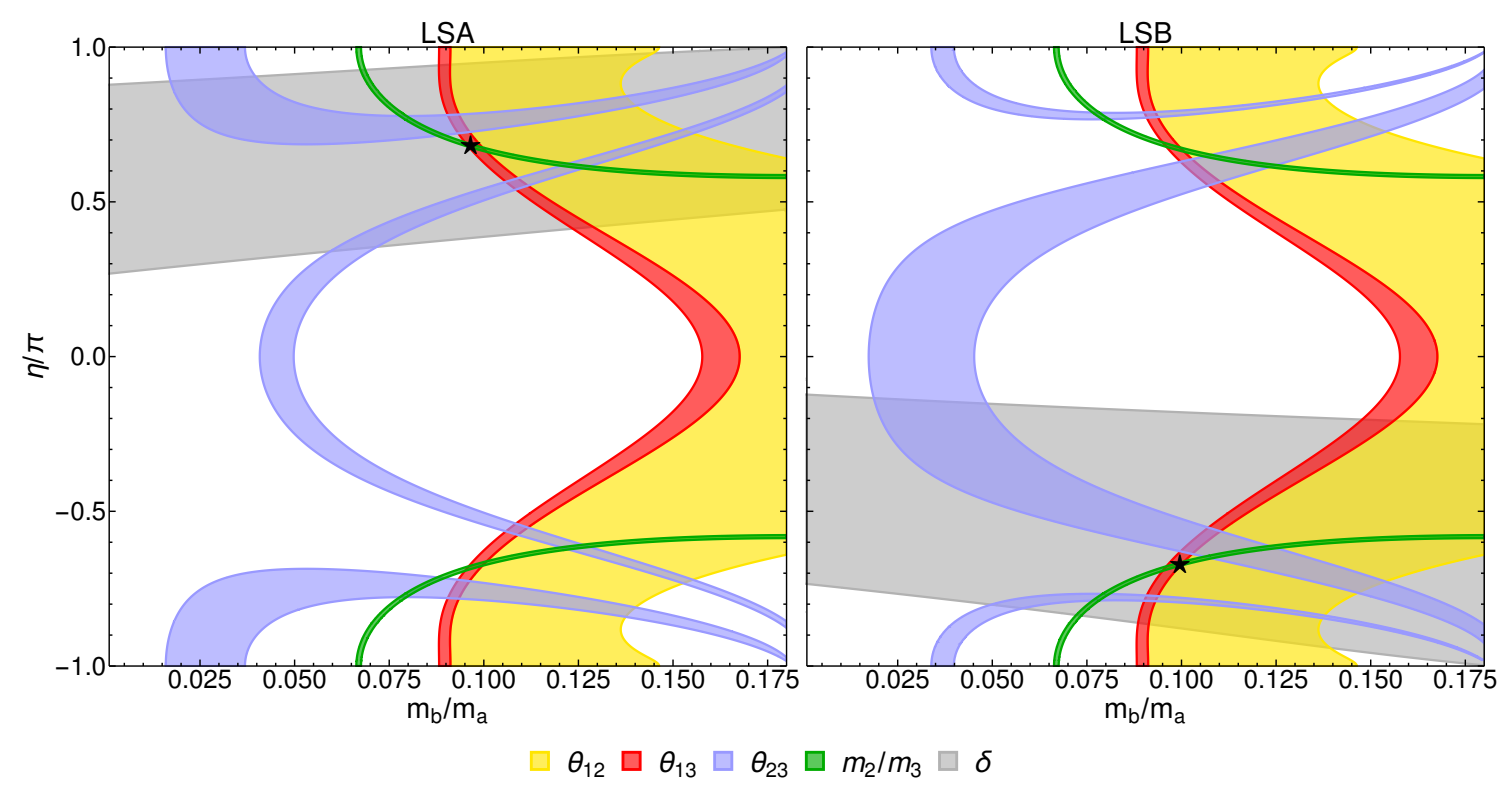

Figure 5. Regions in the $m_{b} / m_{a}-\eta$ plane corresponding to the experimentally determined $1 \sigma$ ranges for all mixing angles, $\delta$ and the ratio of neutrino masses $m_{2} / m_{3}$ for LSA (left panel) and LSB (right panel).

some tension with the value of $\theta_{23}$ even when allowing $\eta$ to vary. As with the case with $\eta$ fixed, this tension exists only at the $1 \sigma$ level, where close to maximal $\theta_{23}$ is excluded.

\subsection{Fitting LS models to global fit data}

In order to provide a more concrete measure of the agreement between the predictions of the model and existing data, as well as to make further predictions of the less well measured parameters, we have performed a $\chi^{2}$ fit to the four cases discussed above: LSA and LSB with $\eta$ fixed and free. As a proxy for the full data sets of previous experiments, our fits use the results of the NuFIT 3.0 global analysis [46]. This analysis combines the latest results (as of fall 2016) of solar, atmospheric, long baseline accelerator, and long, medium and short baseline reactor neutrino experiments, to obtain a combined fit to the six standard neutrino oscillation parameters. We use the $\chi^{2}$ data provided by $\mathrm{NuFIT}^{7}$ for the case where normal mass ordering is assumed, combining both the $1 \mathrm{D} \chi^{2}$ data for each mixing parameter with the $2 \mathrm{D} \chi^{2}$ data to include correlations between parameter measurements

$$
\chi_{\mathrm{Fit}}^{2}(\Theta)=\sum_{\theta_{i} \in \Theta} \chi_{1 \mathrm{D}}^{2}\left(\theta_{i}\right)+\sum_{\theta_{i} \neq \theta_{j} \in \Theta}\left(\chi_{2 \mathrm{D}}^{2}\left(\theta_{i}, \theta_{j}\right)-\chi_{1 \mathrm{D}}^{2}\left(\theta_{i}\right)-\chi_{1 \mathrm{D}}^{2}\left(\theta_{j}\right)\right)
$$

where the first sum in this expression combines each of the 1D $\chi^{2}$ data into a first approximation of the full $6 \mathrm{D} \chi^{2}$ while the second sum provides corrections to this coming from the $2 \mathrm{D}$ correlations between each pair of parameters.

\footnotetext{
${ }^{7}$ We have used the full data for one- and two-dimensional $\Delta \chi^{2}$ projections of the NuFIT analysis, available at http://www.nu-fit.org.
} 


\begin{tabular}{|lccccc|}
\hline & \multicolumn{2}{c}{ LSA } & \multicolumn{2}{c}{ LSB } & NuFIT 3.0 \\
& $\eta$ free & $\eta$ fixed & $\eta$ free & $\eta$ fixed & global fit \\
\hline$m_{a}[\mathrm{meV}]$ & 27.19 & 26.74 & 26.95 & 26.75 & \\
$m_{b}[\mathrm{meV}]$ & 2.654 & 2.682 & 2.668 & 2.684 & - \\
$\eta[\mathrm{rad}]$ & $0.680 \pi$ & $2 \pi / 3$ & $-0.673 \pi$ & $-2 \pi / 3$ & \\
\hline$\theta_{12}\left[^{\circ}\right]$ & $34.36_{-0.02}^{+0.03}$ & $34.33_{-0.01}^{+0.01}$ & $34.35_{-0.03}^{+0.03}$ & $34.33_{-0.03}^{+0.03}$ & $33.72_{-0.76}^{+0.79}$ \\
$\theta_{13}\left[^{\circ}\right]$ & $8.46_{-0.11}^{+0.13}$ & $8.60_{-0.05}^{+0.05}$ & $8.54_{-0.17}^{+0.12}$ & $8.60_{-0.11}^{+0.13}$ & $8.46_{-0.15}^{+0.14}$ \\
$\theta_{23}\left[^{\circ}\right]$ & $45.03_{-0.45}^{+0.44}$ & $45.71_{-0.05}^{+0.05}$ & $44.64_{-0.41}^{+0.63}$ & $44.28_{-0.11}^{+0.12}$ & $41.5_{-1.1}^{+1.3}$ \\
$\delta\left[^{\circ}\right]$ & $-89.9_{-2.0}^{+1.9}$ & $-86.9_{-0.2}^{+0.2}$ & $-91.6_{-1.8}^{+2.8}$ & $-93.1_{-0.5}^{+0.5}$ & $-71_{-51}^{+38}$ \\
$\Delta m_{21}^{2}\left[10^{-5} \mathrm{eV}^{2}\right]$ & $7.499_{-0.131}^{+0.162}$ & $7.379_{-0.070}^{+0.064}$ & $7.447_{-0.129}^{+0.192}$ & $7.390_{-0.152}^{+0.150}$ & $7.49_{-0.17}^{+0.19}$ \\
$\Delta m_{31}^{2}\left[10^{-3} \mathrm{eV}^{2}\right]$ & $2.500_{-0.029}^{+0.027}$ & $2.510_{-0.019}^{+0.018}$ & $2.500_{-0.031}^{+0.034}$ & $2.512_{-0.041}^{+0.039}$ & $2.526_{-0.037}^{+0.039}$ \\
\hline$\Delta \chi^{2} /$ d.o.f & $4.1 / 3$ & $5.6 / 4$ & $3.9 / 3$ & $4.5 / 4$ & - \\
\hline
\end{tabular}

Table 1. Results of our fit of existing data to LSA and LSB with $\eta$ left free and for $\eta=\frac{2 \pi}{3}$ for LSA and $\eta=-\frac{2 \pi}{3}$ for LSB. The best fit values are given for both the input parameters, $m_{a}, m_{b}$ and $\eta$, and the output parameters, $\theta_{i j}, \Delta m_{i j}^{2}$ and $\delta$, together with the $\Delta \chi^{2} /$ degrees of freedom for the best fit. For the output parameters, errors are also given corresponding to the $1 \sigma$ ranges of our fit. The results of the NuFIT 3.0 (2016) global fit to standard neutrino mixing for the normal ordering case are shown for comparison.

We then apply this result first to the standard mixing case, then to the LS model case as follows:

- For the case of standard mixing $\Theta=\Theta_{\mathrm{PMNS}} \equiv\left\{\theta_{12}, \theta_{13}, \theta_{23}, \Delta m_{21}^{2}, \Delta m_{31}^{2}, \delta\right\}$ and we simply combine the NuFIT 3.0 results as shown above, in order to include correlations, and use it to calculate $\chi^{2}\left(\Theta_{\text {PMNS }}\right) \equiv \chi_{\text {Fit }}^{2}(\Theta)$ for this case.

- For the LS model we use instead $\Theta=\Theta_{L S} \equiv\left\{m_{a}, m_{b}, \eta\right\}$ (or $\Theta_{\mathrm{LS}}=\left\{m_{a}, m_{b}\right\}$ when fitting with $\eta$ fixed), which is then minimised over the LS parameter space using the analytic relations to calculate standard mixing parameters from LS parameters, and hence calculate $\chi^{2}\left(\Theta_{\mathrm{LS}}\right) \equiv \chi_{\text {Fit }}^{2}(\Theta)$ for this case.

Our test statistic for a particular LS model is then given by:

$$
\sqrt{\Delta \chi^{2}}=\sqrt{\min _{\Theta_{\mathrm{LS}}}\left[\chi^{2}\left(\Theta_{\mathrm{LS}}\right)\right]-\min _{\Theta_{\mathrm{PMNS}}}\left[\chi^{2}\left(\Theta_{\mathrm{PMNS}}\right)\right]} .
$$

We have verified through Monte-Carlo calculations that Wilks' theorem [74] holds for this statistic, i.e. it is approximately distributed according to a chi-squared distribution.

The best fit LSA and LSB points for fits with $\eta$ left free or with $\eta$ fixed at $\frac{2 \pi}{3}$ are given in table 1 together with errors on the predicted parameters corresponding to the $1 \sigma$ allowed ranges. The number of degrees of freedom (d.o.f.) is either 3 or 4 , which is just 

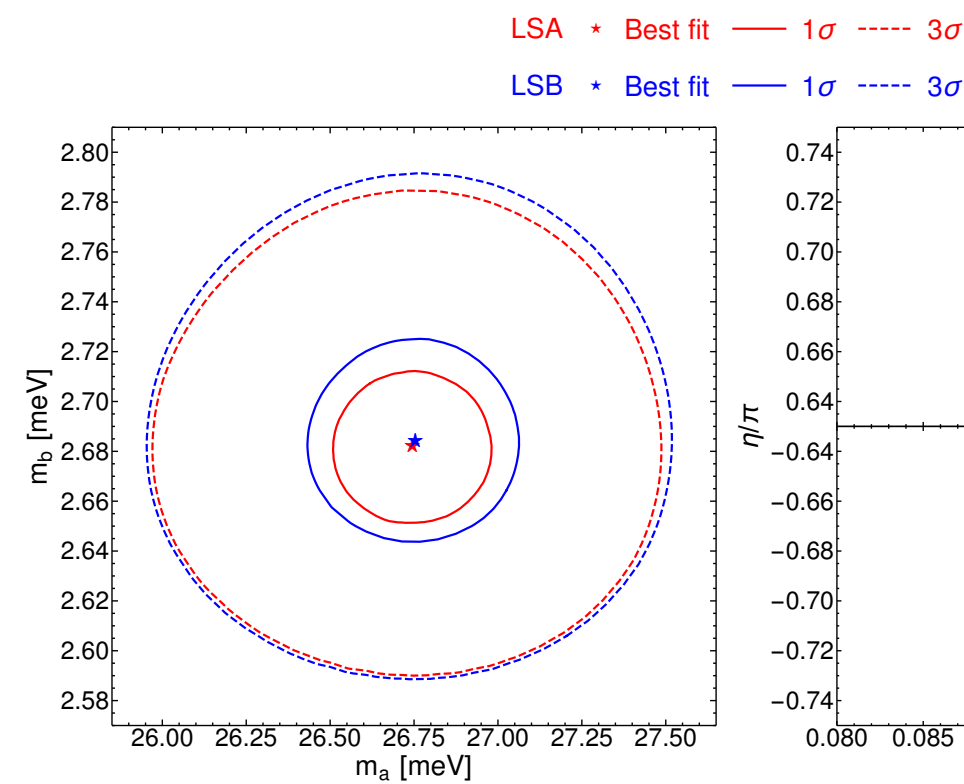

Figure 6. Results of the fits to LS of the NuFIT 3.0 (2016) global neutrino oscillation data. Left: LS fit with fixed $\eta=2 \pi / 3(\eta=-2 \pi / 3)$ for LSA (LSB). Right: LS fit with $\eta$ as a free parameter.

the difference between the number of observables (which we take to be the parameters in $\Theta_{\text {PMNS }}$ ) and the number of LS parameters (namely the parameters in $\Theta_{\mathrm{LS}}$, which is either 3 or 2 , depending on whether $\eta$ is free or fixed). For LSA we find a best fit with $\Delta \chi^{2}=4.1$ (3 degrees of freedom) with $\eta$ free and $\Delta \chi^{2}=5.6$ (4 degrees of freedom) fixing $\eta=\frac{2 \pi}{3}$, while for LSB we find better fits, with $\Delta \chi^{2}=3.9$ (3 degrees of freedom) and $\Delta \chi^{2}=4.5$ ( 4 degrees of freedom) for $\eta$ free and $\eta=-\frac{2 \pi}{3}$ respectively.

Figure 6 shows the best fit points with $1 \sigma$ and $3 \sigma$ contours of the fits in the $m_{a}-m_{b}$ plane for fixed $\eta$ and in the $r-\eta$ plane for free $\eta$. The significance at which a LS model is allowed is determined from the distribution of the $\Delta \chi^{2}$ test statistic, where $N \sigma$ has been calculated assuming the that Wilks' theorem applies. Note that despite LSA predicting values of $\theta_{23}$ which lie outside its individual $1 \sigma$ range reported by NuFIT 3.0, there are still regions not excluded at $1 \sigma$. This is due to the high predictivity of the model; by predicting many parameters from few input parameters there is a greater chance that one of these may lie outside its experimentally determined range. Statistically, this comes from the increased number of degrees of freedom of the $\chi^{2}$-distribution which approximates our test statistic $\Delta \chi^{2}$.

Our fit can also be used to identify the regions of standard neutrino mixing parameter space predicted by LS, once existing data has been taken into account. This corresponds to mapping the regions of LS input parameter space allowed by our fit onto the standard mixing parameter space. Figure 7 shows the predictions of LS (for the fixed $\eta$ case) in the planes made from each pair of mixing angles and $\delta$. Since these values all depend only on the single parameter $r$, the predictions of LS form lines of allowed solutions in each plane, corresponding to sum-rules between the oscillation parameters. For example, figure 7a corresponds to the TM1 sum rule in eq. (2.6), while figures $7 \mathrm{~b}$ to $7 \mathrm{f}$ correspond to those in 

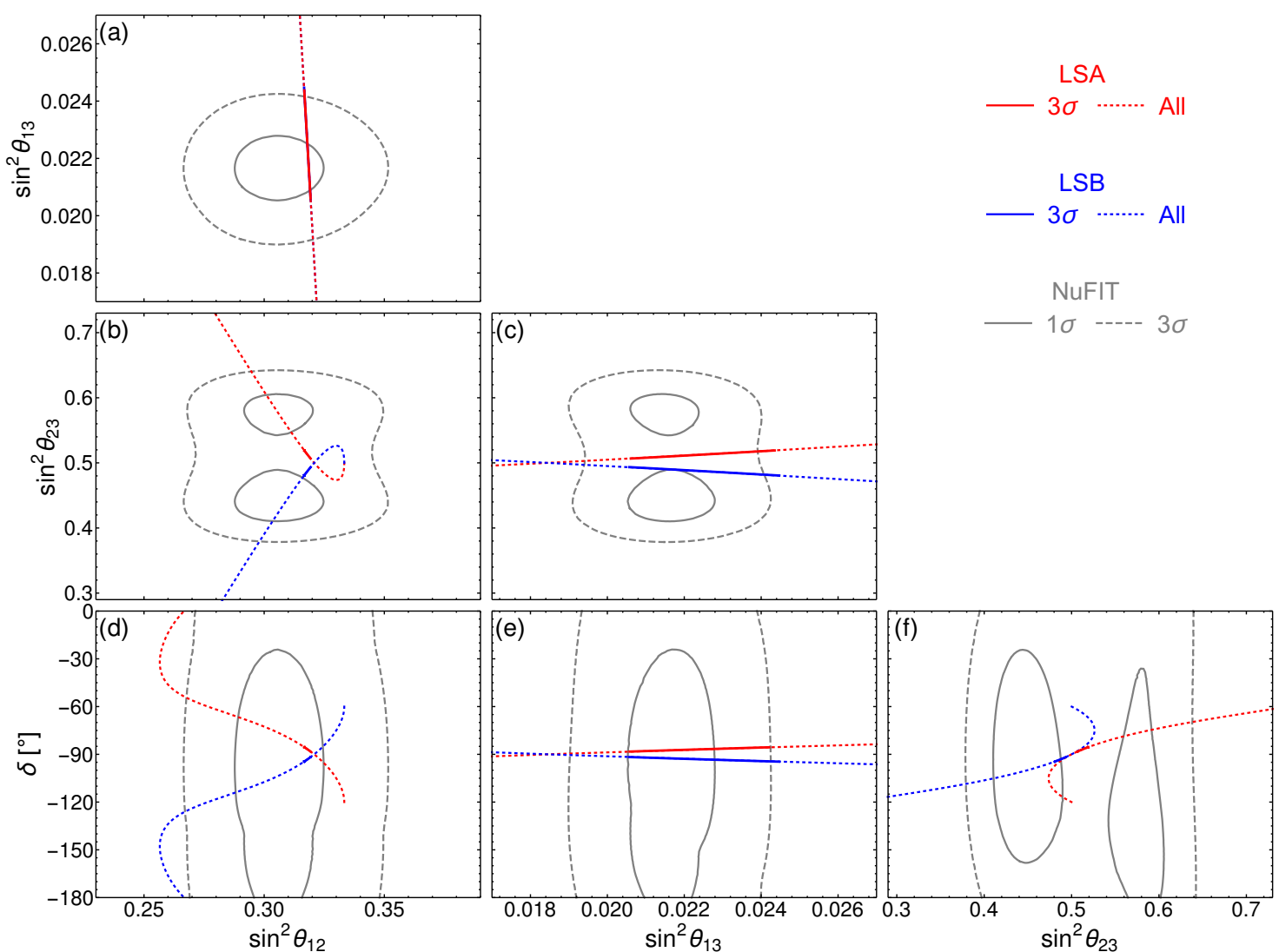

Figure 7. Allowed values for LSA (red) and LSB (blue) with $\eta=2 \pi / 3$ and $\eta=-2 \pi / 3$ respectively. The dotted lines labelled "All" show all possible values allowed by the model, corresponding to the solutions of the sum rules in eqs. (2.6) and (A.6) or combinations thereof without any constraints from experimental data, with the solid segments showing those values allowed experimentally at $3 \sigma$. Also shown are the $1 \sigma$ (solid) and $3 \sigma$ (dashed) regions from the NuFIT 3.02016 global fit (grey).

eq. (A.6) or to combinations of these sum rules. It can be seen that very strong restrictions are placed on the allowed values of the less well measured parameters, $\theta_{12}, \theta_{23}$ and $\delta$. For the remaining angle, $\theta_{13}$, around two thirds of the NuFIT 3.0 range remains viable in LS.

Figure 8 shows the allowed regions of parameter space for pairs of variables including the mass-squared differences. In these plots, as the mass-squared differences can depend on both $m_{a}$ and $m_{b}$ independently, we see regions of allowed values instead of lines. For each of these planes, any point will fully determine both input parameters $m_{a}$ and $m_{b}$, and so these contours correspond exactly to the equivalent regions shown in figure 6 . In addition to the tight constraints on $\theta_{12}, \theta_{23}$ and $\delta$ already mentioned, in figures $8 \mathrm{~b}$ and $8 \mathrm{e}$ it can be seen that the allowed range of $\theta_{13}$ is correlated with that of both $\Delta m_{21}^{2}$ and $\Delta m_{31}^{2}$, suggesting that combining future measurements of these parameters could provide a better probe of LS than the individual parameter measurements alone. The ability of future experiment to exclude the model then depends on both the predictions of the model seen here, combined with the sensitivity of experiments to measurements of the parameters in the region of interest predicted by LS, which is the focus of the next section. 

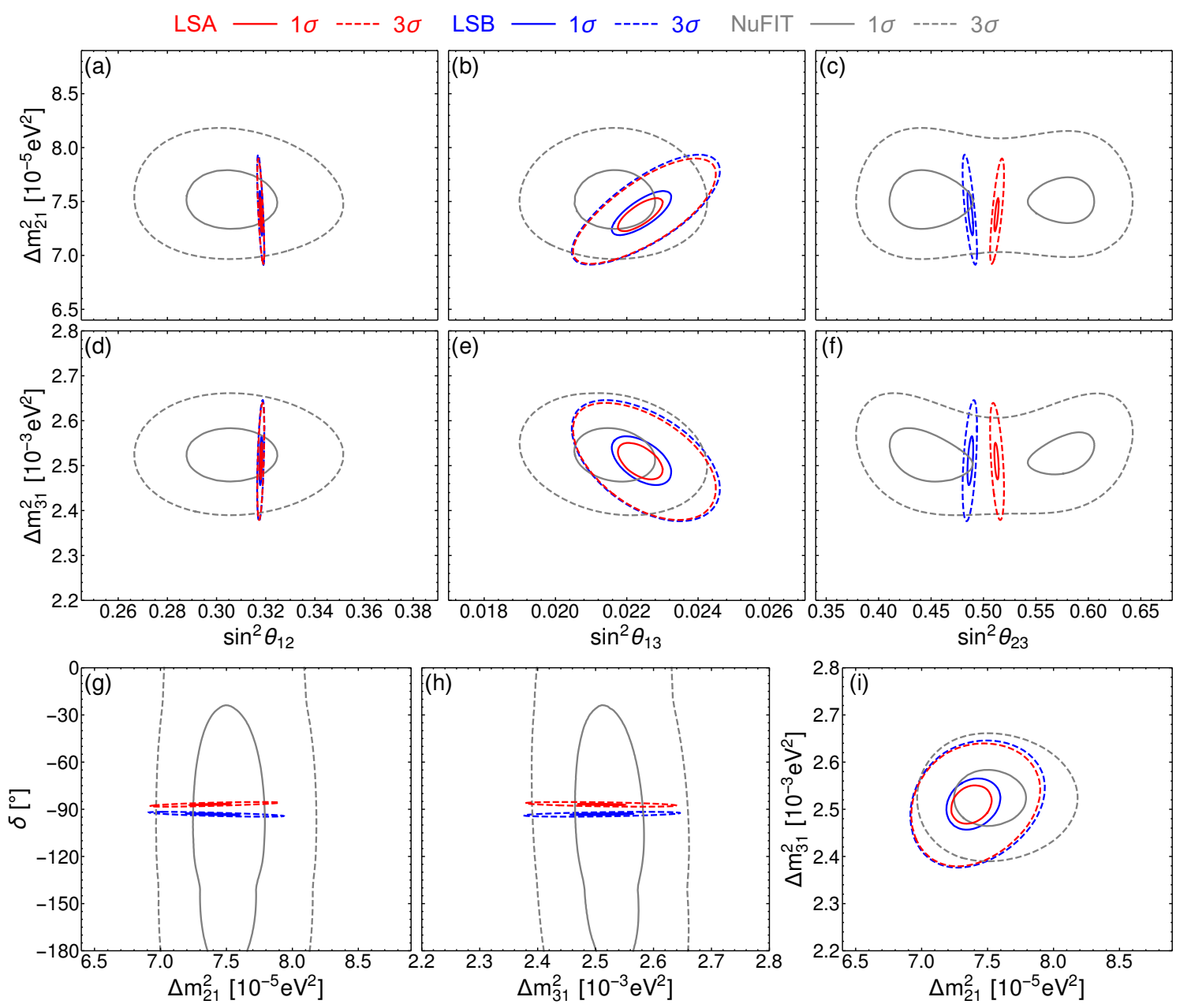

Figure 8. Allowed $1 \sigma$ (solid) and $3 \sigma$ (dashed) regions for LSA (red) and LSB (blue) with $\eta=2 \pi / 3$ and $\eta=-2 \pi / 3$ respectively. Also shown are the current allowed regions from the NuFIT 3.02016 global fit (grey).

\section{Sensitivity of future experiments}

In order to understand the potential for future experiments to exclude the LS models, we have performed simulations of a combination of accelerator and reactor experiments, modelling the experimental data expected over the next two decades. We have used the General Long Baseline Experiment Simulator (GLoBES) libraries [68, 69] to simulate future experiments and to fit the simulated data to both standard mixing and the LS models. In all our simulations we assume that the mass ordering is known to be normal ordering, as this is a requirement of the LS models; a measurement of inverted ordering would immediately exclude the models.

\subsection{Future neutrino oscillation experiments}

Our combination of experiments include detailed simulations of the T2HK and DUNE longbaseline accelerator experiments, which aim to provide precision measurements of $\Delta m_{31}^{2}$, 
$\theta_{23}$ and $\delta$, together with basic constraints on $\theta_{13}$ from the Daya Bay short baseline reactor experiment and on $\theta_{12}$ and $\Delta m_{21}^{2}$ from the JUNO and RENO-50 medium baseline reactor experiments. We will now briefly recap the salient features of these experiments and our treatment of them.

T2HK. The Tokai to Hyper-Kamiokande (T2HK) experiment is a proposed long-baseline accelerator neutrino experiment using the Hyper-Kamiokande detector, a megatonne scale water Cherenkov detector to be constructed near to the Super-Kamiokande detector in Kamioka, Japan [61]. The standard design is for two tanks to be built, each with $258 \mathrm{kt}$ (187 kt) of total (fiducial) volume. The tanks are to be built in a staged process with the second tank constructed and commissioned after the first, such that the second begins to take data six years after the first. The water Cherenkov technique is capable of detecting the (anti-)muons and electrons (positrons) produced in (anti-)neutrino interactions, with the ability to distinguish the charged leptons' flavours but not their charge. The detector would be used to observe neutrinos from (amongst other sources) an upgraded version of the T2K neutrino beam produced at J-PARC in Tokai, $L=295 \mathrm{~km}$ from the detector. The 1.3 MW beam, produced from a $30 \mathrm{GeV}$ protons, is directed $2.5^{\circ}$ away from the detector in order to provide a narrow energy spectrum at the far detector peaked around the first atmospheric neutrino oscillation maximum for $\Delta m_{31}^{2} \sim 2.5 \times 10^{-3} \mathrm{eV}^{2}$ and $E=0.6 \mathrm{GeV}$. Either $\nu_{\mu}$ or $\bar{\nu}_{\mu}$ can be produced as the principle component of the beam, such that the oscillation probabilities $P\left(\nu_{\mu} \rightarrow \nu_{e}\right), P\left(\bar{\nu}_{\mu} \rightarrow \bar{\nu}_{e}\right), P\left(\nu_{\mu} \rightarrow \nu_{\mu}\right)$ and $P\left(\bar{\nu}_{\mu} \rightarrow \bar{\nu}_{\mu}\right)$ can all be measured. While the main goal of T2HK is to search for CP symmetry violation by observing a non-CP conserving value of $\delta$, precision measurements of $\theta_{23}$ and the magnitude of $\Delta m_{31}^{2}$ will also be made [62].

DUNE. The Deep Underground Neutrino Experiment [63] (DUNE) is a proposed longbaseline accelerator experiment, which differs from the T2HK experiment through its longer baseline and higher energy wide-band beam. The experiment will use a new neutrino beam sourced at Fermilab, directed towards a large liquid argon detector in Sanford, $L=1300 \mathrm{~km}$ from the beam source. The $40 \mathrm{kt} \mathrm{LArTPC}$ detector is able to detect both the charged leptons and the hadrons produced from muon and electron (anti-)neutrino interactions, with strong particle identification and energy reconstruction capabilities. The standard design is for a $1.07 \mathrm{MW} \nu_{\mu}$ or $\bar{\nu}_{\mu}$ beam produced from $80 \mathrm{GeV}$ protons, with an on-axis design to produce a wide energy spectrum spanning $E=0.5$ to $5 \mathrm{GeV}$, allowing observations of the $\nu_{e}$ appearance spectrum around the first atmospheric neutrino oscillation maximum for $\Delta m_{31}^{2} \sim 2.5 \times 10^{-3} \mathrm{eV}^{2}$. While measuring the same oscillation channels as T2HK, the wider band beam with longer baseline provides complementary information on the value of $\delta$ as well as measurements of $\theta_{23}$ and, due to the matter effects from the longer baseline, both the sign and the magnitude of $\Delta m_{31}^{2}$ [64].

Short baseline reactor experiments. By observing the oscillations of the $\bar{\nu}_{e}$ produced in nuclear reactors, short baseline reactor neutrino experiments are able to measure the mixing angle $\theta_{13}$ with particularly high accuracy. The Daya Bay experiment [65] currently has the most precise measurement of this parameter with the aim to achieve a precision on 


\begin{tabular}{|lll|}
\hline Experiment & Parameter & Precision \\
\hline Short baseline reactor & $\sin ^{2} \theta_{13}$ & $3 \%$ \\
Medium baseline reactor & $\sin ^{2} \theta_{12}$ & $0.5 \%$ \\
Medium baseline reactor & $\Delta m_{21}^{2}$ & $0.5 \%$ \\
\hline
\end{tabular}

Table 2. Precision of oscillation parameter measurements made by reactor experiments which we have used as constraints in our simulations.

$\sin ^{2} \theta_{13}$ of better than $3 \%$ [66]. The experiment measures anti-neutrinos produced in six nuclear reactors in south China. A total of eight $20 \mathrm{t}$ liquid scintillator detectors are used; two are located at each of two near detector sites and four at a far detector site $L=1.5$ to $1.9 \mathrm{~km}$ from the reactors near the first atmospheric neutrino oscillation maximum for $\Delta m_{31}^{2} \sim 2.5 \times 10^{-3} \mathrm{eV}^{2}$, given the low nuclear energy of the neutrino beam $E \sim$ few $\mathrm{MeV}$. Results of the Double Chooz [72] and RENO [71, 73] short baseline reactor experiments also contribute to the precision obtained on $\theta_{13}$ combined with the Daya Bay result. Although DUNE and T2HK will also measure this parameter with high precision, the measurement of the short baseline reactor programme by that time is expected to be at least as precise, and will provide a measurement independent of the other parameters which influence the appearance channel at long-baseline accelerator experiments.

Medium baseline reactor experiments. The Jiangmen Underground Neutrino Observatory [67] (JUNO) and the future plans of the Reactor Experiment for Neutrino Oscillation (RENO-50) [71] are medium baseline reactor neutrino experiments which, like the Daya Bay experiment, will observe the oscillations of electron anti-neutrinos produced in nuclear reactors. The JUNO experiment will use a $20 \mathrm{kt}$ liquid scintillator detector approximately $L=53 \mathrm{~km}$ from two planned nuclear reactors in southern China, while RENO-50 will use an $18 \mathrm{kt}$ liquid scintillator detector approximately $L=50 \mathrm{~km}$ from a nuclear reactor in South Korea. Given the low nuclear energy of the neutrino beam $E \sim$ few $\mathrm{MeV}$, these longer baselines correspond to the first solar neutrino oscillation maximum for $\Delta m_{21}^{2} \sim 7.5 \times 10^{-5} \mathrm{eV}^{2}$, where the higher frequency atmospheric oscillations appear as wiggles. Thus the longer baseline than at Daya Bay gives greatest sensitivity to a different set of oscillation parameters, in particular $\theta_{12}$ and $\Delta m_{21}^{2}$. The precision on the measurements of both $\sin ^{2} \theta_{12}$ and $\Delta m_{21}^{2}$ is expected to reach $0.5 \%[67,71]$.

Details of experimental simulation. We have used complete simulations of the latest designs for both DUNE and T2HK where we have assumed both experiments run for 10 years. Full details of the GLoBES implementations we have used can be found in [70]. For the short and medium baseline reactor experiments, we have included basic constraints on the values of $\sin ^{2} \theta_{13}, \sin ^{2} \theta_{12}$ and $\Delta m_{21}^{2}$. Since these measurements are expected to be approximately independent of other parameters we have implemented these constraints as simple Gaussian measurements with a mean of the true simulated value and error as given in table 2 . 


\subsection{Statistical method}

To determine the statistical significance with which the LS model could be excluded based on simulated data, we perform a minimum- $\chi^{2}$ fit to both standard three neutrino mixing and to the LS model. As in section 3.3, for the case of standard mixing we use $\Theta=$ $\Theta_{\text {PMNS }} \equiv\left\{\theta_{12}, \theta_{13}, \theta_{23}, \Delta m_{21}^{2}, \Delta m_{31}^{2}, \delta\right\}$, while for LS we use $\Theta=\Theta_{L S} \equiv\left\{m_{a}, m_{b}, \eta\right\}$ (or $\Theta_{\mathrm{LS}}=\left\{m_{a}, m_{b}\right\}$ when fitting with $\eta$ fixed). Our test statistic for the significance to exclude the LS model is then given by

$$
\sqrt{\Delta \chi^{2}}=\sqrt{\min _{\Theta_{\mathrm{LS}}}\left[\chi^{2}\left(\Theta_{\mathrm{LS}}\right)\right]-\min _{\Theta_{\mathrm{PMNS}}}\left[\chi^{2}\left(\Theta_{\mathrm{PMNS}}\right)\right]} .
$$

The significance at which LS is excluded is then determined from the distribution of the $\Delta \chi^{2}$ test statistic; where we give sensitivities in terms of $N \sigma$, this quantity has been calculated assuming the that Wilks' theorem applies. Wilks' theorem states that when comparing nested models, the $\Delta \chi^{2}$ test statistic is a random variable asymptotically distributed according to the $\chi^{2}$-distribution with the number of degrees of freedom equal to the difference in number of free parameters in the models. In this case we treat the LS models, with two or three free parameters, as sub-models of standard neutrino mixing with six free parameters, leading to a $\chi^{2}$-distribution with 4 degrees of freedom when $\eta$ is kept fixed or 3 degrees of freedom when $\eta$ is left as a free parameter. We have verified via MonteCarlo simulations that the distribution of our $\Delta \chi^{2}$ test statistic is well approximated by these distributions.

In applying the above formula, the $\chi^{2}(\Theta)$ is minimised over the parameters $\Theta$ in our fits and is built from three parts;

$$
\chi^{2}(\Theta)=\chi_{\mathrm{LB}}^{2}(\Theta)+\chi_{\mathrm{R}}^{2}(\Theta)+P(\Theta),
$$

with $\chi_{\mathrm{LB}}^{2}(\Theta)$ for the full simulations of the long-baseline experiments DUNE and T2HK, $\chi_{\mathrm{R}}^{2}(\Theta)$ for the constraints from reactor experiments Daya Bay and JUNO, and $P(\Theta)$ for a prior intended to include information from the results of existing experimental measurements.

For the long-baseline experiments we use the statistical model of the GLoBES library [68, 69], where the $\chi_{L B}^{2}(\Theta)$ is a sum of contributions from each of the experiments' channels. The individual contributions are constructed as

$$
\chi_{c}^{2}(\Theta)=\min _{\xi=\left\{\xi_{s}, \xi_{b}\right\}}\left[2 \sum_{i}\left(\eta_{i}(\Theta, \xi)-n_{i}+n_{i} \ln \frac{n_{i}}{\eta_{i}(\Theta, \xi)}\right)+p(\xi, \sigma)\right],
$$

where $\chi_{c}^{2}$ denotes the contribution from a given channel of a given experiment. The sum in this expression is over the $i$ energy bins of the experimental configuration, with simulated true event rates of $n_{i}$ and simulated event rates $\eta_{i}(\Theta, \xi)$ for the hypothesis parameters $\Theta$ and systematic error parameters $\xi$. The systematic errors of the experiments are treated using the method of pulls, parameterized as $\xi_{s}$ for the signal error and $\xi_{b}$ for the background error. These parameters are given Gaussian priors which form the term $p(\xi, \sigma)=\xi_{s}^{2} / \sigma_{s}^{2}+\xi_{b}^{2} / \sigma_{b}^{2}$, where $\sigma=\left\{\sigma_{s}, \sigma_{b}\right\}$ are the sizes of the systematic errors given by the experiment. 
For the reactor experiments we simply assume independent Gaussian measurements such that

$$
\chi_{\mathrm{R}}^{2}=\frac{\left(\sin ^{2} \theta_{13}-\sin ^{2} \overline{\theta_{13}}\right)^{2}}{\sigma_{\theta_{13}}^{2}}+\frac{\left(\sin ^{2} \theta_{12}-\sin ^{2} \overline{\theta_{12}}\right)^{2}}{\sigma_{\theta_{12}}^{2}}+\frac{\left(\Delta m_{21}^{2}-\overline{\Delta m_{21}^{2}}\right)^{2}}{\sigma_{\Delta m_{21}^{2}}^{2}},
$$

where $\overline{\theta_{13}}, \overline{\theta_{12}}$ and $\overline{\Delta m_{21}^{2}}$ are the true parameter values and $\sigma_{\theta_{13}}, \sigma_{\theta_{12}}$ and $\sigma_{\Delta m_{21}^{2}}$ the corresponding experimental measurement uncertainties.

The prior $P(\Theta)$ provides information from existing experimental measurements and is calculated using the results of the NuFIT 3.0 global fit in the same way as our fit in section 3.3, so that $P(\Theta)=\chi_{\text {Fit }}^{2}(\Theta)$ as defined in eq. (3.1).

In all our simulations, the true parameters are taken to be the best-fit values from the appropriate LS fit results given in table 1, except where stated otherwise. During minimisation of $\Delta \chi^{2}$, the test parameters are allowed to vary with no restriction on their values. This means that $m_{a}$ and $m_{b}$ for LS and the mass-squared differences for standard mixing can have any value greater than 0 , with $\eta$ for LS and the mixing angles and phase $\delta$ for standard mixing taking any value from $-\pi$ to $\pi$. However, we found that in call cases in our simulations the parameter values at the $\Delta \chi^{2}$ minimum remained close to the specified true values (for standard mixing parameters) and the physically viable ranges found in the fits of the previous section (for LS parameters).

\subsection{Results}

The sensitivity to exclude either version of the LS model is shown as a function of the true value of each parameter in figure 9 , for true values, with the range selected along the horizontal axes to be that given by the currently allowed at $3 \sigma$ by the latest NuFIT 3.0 global fit. In each case, the parameters not shown are assumed to take their best-fit values from the fit to LS described in section 3.3.

From the upper panels in figure 9 , we see that $\theta_{12}, \theta_{23}$ and $\delta$ provide the strongest tests of the model, with there only being a relatively small portion of the presently allowed true parameter space where the model would not be excluded. This is due to the strong predictions of these parameters by the LS models, as discussed in section 3.1. Note that these parameters are those that will be measured most precisely by the three next-generation experiments used in our simulations, JUNO, DUNE and T2HK. For these three parameters, the effect of allowing $\eta$ to vary does not much change the sensitivity, other than the additional solution (currently disfavoured by experiment) with $\delta=+90^{\circ}$ which occurs when changing the sign of $\eta$. For $\theta_{12}$ in particular there is no effect of allowing $\eta$ to vary. This is due to the sum rule in eq. (2.6) which relates $\theta_{12}$ with $\theta_{13}$ independently from the value of $\eta$; the precise measurement of $\theta_{13}$ then fixes the value of $\theta_{12}$ to a narrow range such that a measurement of $\theta_{12}$ outside of this would exclude the LS model regardless of the LS parameter values. Similarly the precise measurements of $\theta_{13}, \Delta m_{21}^{2}$ and $\Delta m_{31}^{2}$ strongly constrain the magnitude (but not sign) of $\eta$, so that the LS allowed regions of the other variables are not significantly changed when $\eta$ is allowed to vary, with the noted exception that changing the sign of $\eta$ allows the sign of $\delta$ to also change. 

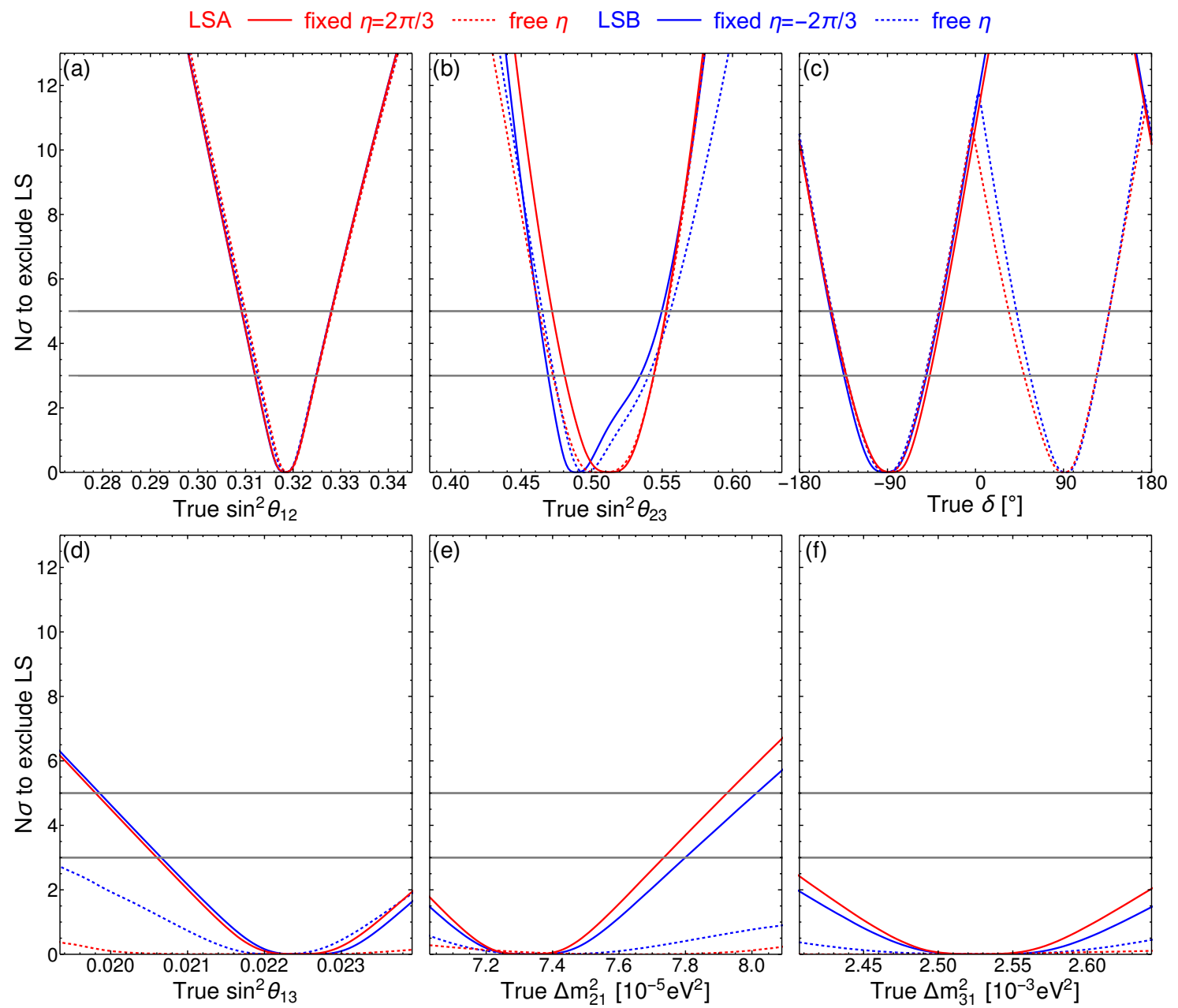

Figure 9. The predicted sensitivity of future experiments to excluding LSA (red) and LSB (blue), shown as a function of the true value of each parameter. Solid curves correspond to the case with $\eta$ fixed at $\eta=\frac{2 \pi}{3}$ for LSA or $\eta=-\frac{2 \pi}{3}$ for LSB, while dashed curves correspond to the case with $\eta$ left free. The ranges of true parameters shown in the plots corresponds to the current three sigma allowed NuFIT 3.0 regions.

From the lower panels in figure 9, we see that the sensitivity to exclude LS from measurements of $\theta_{13}, \Delta m_{21}^{2}$ or $\Delta m_{31}^{2}$ is much less than for the other three parameters and the sensitivity is also significantly reduced when allowing $\eta$ to vary. By the converse argument to that used above, this is due to these three parameter measurements driving the fit to $m_{a}$ and $m_{b}$ (and $\eta$ ), and so a measurement of these parameters will tend to move the fitted LS parameter values rather than exclude the model, particularly when fitting the extra free parameter $\eta$. However, a particularly small measurement of $\theta_{13}$ or particularly large measurement of $\Delta m_{21}^{2}$, relative to their current allowed range of values, may still exclude the fixed $\eta$ version of the models.

The results shown in figure 9 show only the dependence of the significance to exclude LS on the true value of each variable individually. However, the sensitivity will generally 

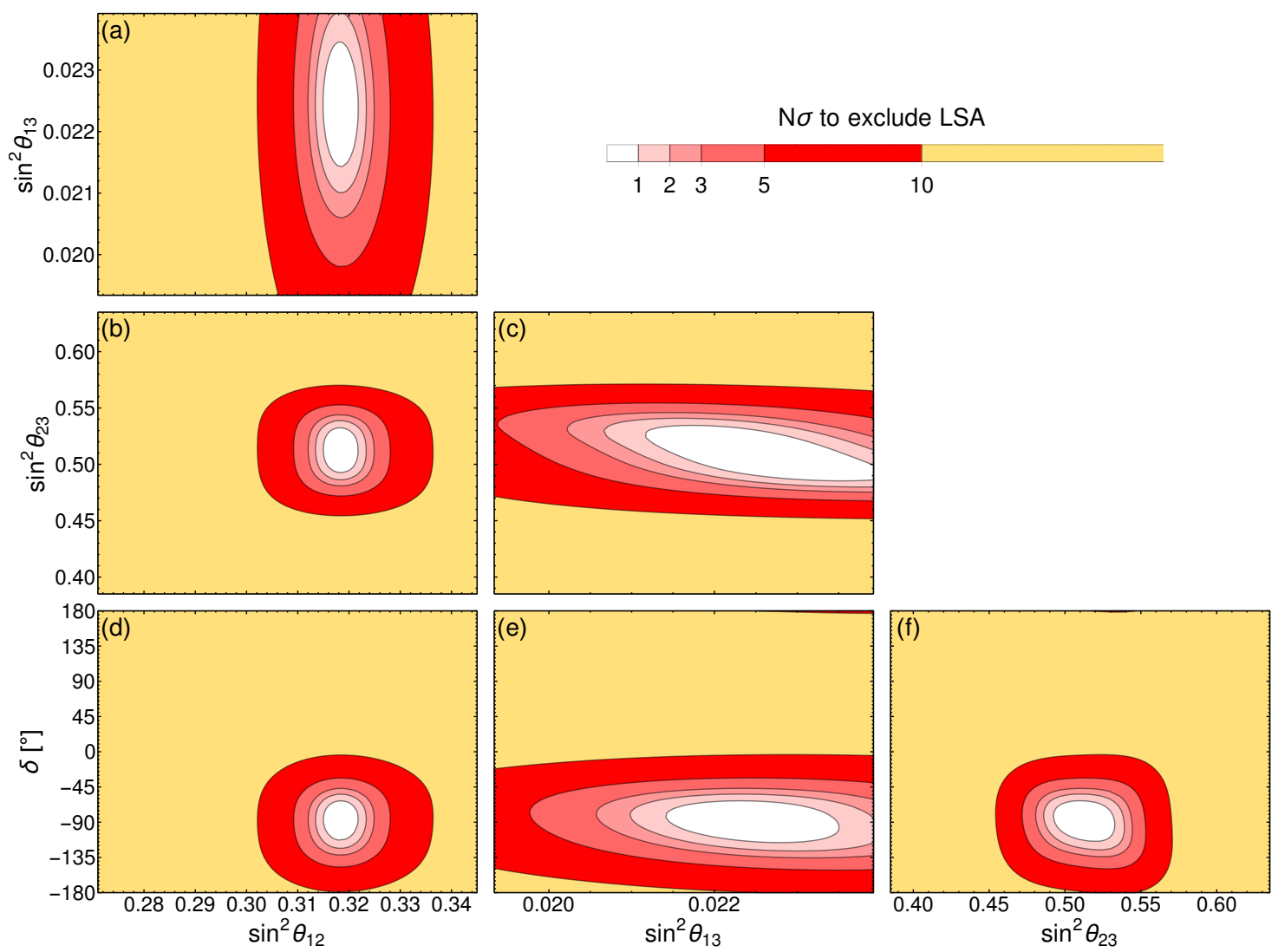

Figure 10. The predicted sensitivity of future experiments to excluding LSA, with $\eta$ fixed at $\eta=\frac{2 \pi}{3}$, shown as a function of each pair of true parameters. The ranges of true parameters shown in the plots corresponds to the current three sigma allowed NuFIT 3.0 regions.

have a strong dependence on the true values of the other parameters. The significance to exclude the LS models depending on the true values of each pair of variables, for the cases where $\eta$ is kept fixed, is shown in Figures 10 and 12 for LSA and in figures 11 and 13 for LSB.

Each panel of figures 10 and 11 includes two dimensionless variables (i.e. angle or phase) which both depend only on the ratio of LS input parameters $r=m_{b} / m_{a}$, and so, in a LS model, a measurement of any one of these parameters corresponds to a measurement of $r=m_{b} / m_{a}$ (see figure 3). Combining two of these parameter measurement therefore give two measurements of $r=m_{b} / m_{a}$, with any conflict between them providing strong evidence to exclude the model. For this reason the significance to exclude the models is close to being simply the combined significance from individual measurements implied by figure 9 .

By contrast, each panel of figures 12 and 13 shows the results for the pairs of variables including at least one dimensionful mass-squared difference. Here we can see in figures 12b, 12e and 12i for LSA, and in figures 13b, 13e and 13i for LSB, there is a strong correlation between the measurements of $\theta_{13}, \Delta m_{21}^{2}$ and $\Delta m_{31}^{2}$. This shows clearly that, although individual measurements of these parameters cannot exclude a LS model (since the parameters of the LS model could be adjusted to accommodate any of them individually) a combined measurement of two of them could serve to exclude the model. This is the 

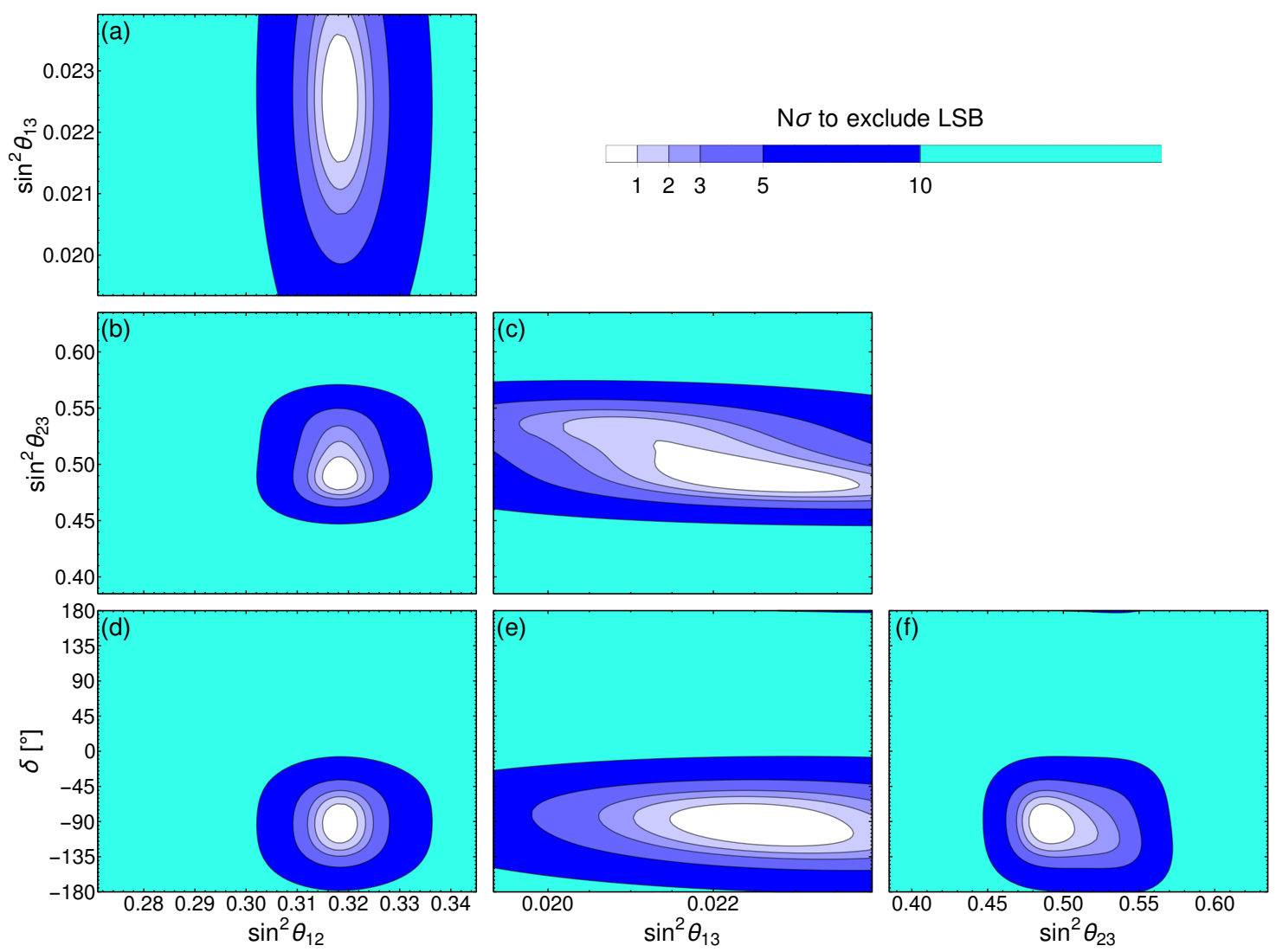

Figure 11. The predicted sensitivity of future experiments to excluding LSB, with $\eta$ fixed at $\eta=-\frac{2 \pi}{3}$, shown as a function of each pair of true parameters. The ranges of true parameters shown in the plots corresponds to the current three sigma allowed NuFIT 3.0 regions.

reason for presenting these combined sensitivity plots. Of the three parameters for which such combined measurements provide the strongest test of the model, each pair includes measurements from different experiments, with $\theta_{13}$ coming mainly from the short-baseline reactor measurement such as Daya Bay, $\Delta m_{21}^{2}$ from the medium-baseline reactor measurement such as JUNO, and $\Delta m_{31}^{2}$ from the long-baseline accelerator measurement such as DUNE and T2HK. This demonstrates a strong synergy between all these experiments in attempts to exclude the LS models.

\section{Conclusion}

In this paper, we have investigated the ability to probe one of the most predictive viable neutrino mass and mixing models with future neutrino oscillation experiments: the Littlest Seesaw. The LS models work within the framework of the Type I seesaw mechanism, using two right-handed neutrinos to generate the left-handed neutrino masses. Combined with constraints from flavour symmetries, the neutrino mixing angles and phases can be predicted from a small number of parameters; in its most constrained form all neutrino masses, angles, and phases are determined from just two input parameters. In fact, we have 


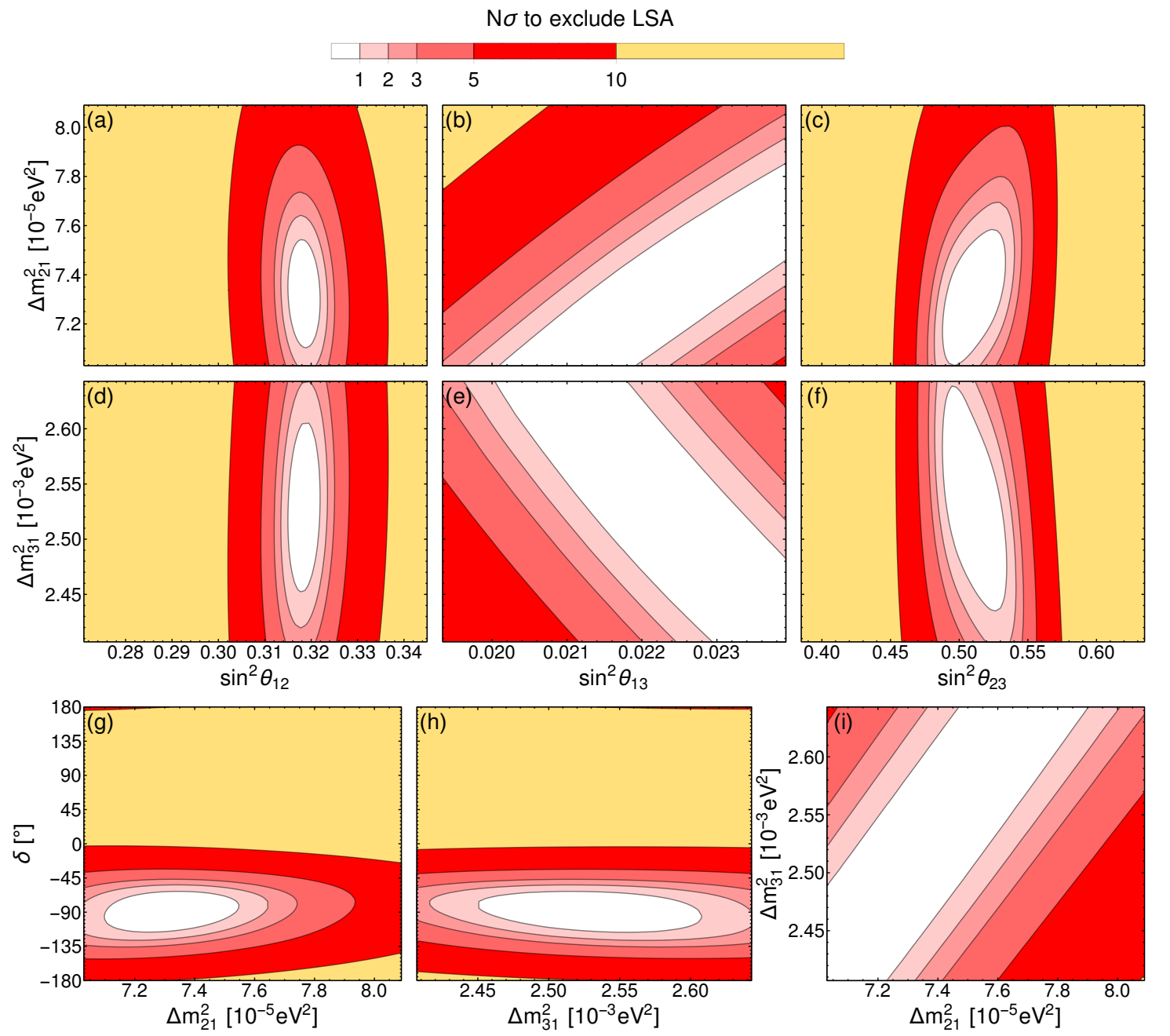

Figure 12. The predicted sensitivity of future experiments to excluding LSA, with $\eta$ fixed at $\eta=\frac{2 \pi}{3}$, shown as a function of each pair of true parameters. The ranges of true parameters shown in the plots corresponds to the current three sigma allowed NuFIT 3.0 regions.

shown that while the neutrino masses depend on the two mass parameters independently, the mixing angles and phases depend only on a single dimensionless quantity, the ratio of these two input parameters.

We have studied two versions of this model (LSA and LSB) which use different flavour symmetries to enforce constraints which result in different permutations of the second and third rows and columns of the neutrino mass matrix, leading to different predictions for the octant of $\theta_{23}$. Using the results of a recent global fit of neutrino oscillation experiments, we have found that both versions can well accommodate the parameter values as measured by experiment, with the greatest tension on the value of $\theta_{23}$ at the $1 \sigma$ level. The prediction of LS is very close to the maximal mixing value with experimental results from $\mathrm{NO} \nu \mathrm{A}$ suggesting a more non-maximal value, while results from $\mathrm{T} 2 \mathrm{~K}$ still consistent with a maximal value of $\theta_{23}$. We find that the LSB version, predicting a value of $\theta_{23}$ in the lower octant, to be slightly preferred. 

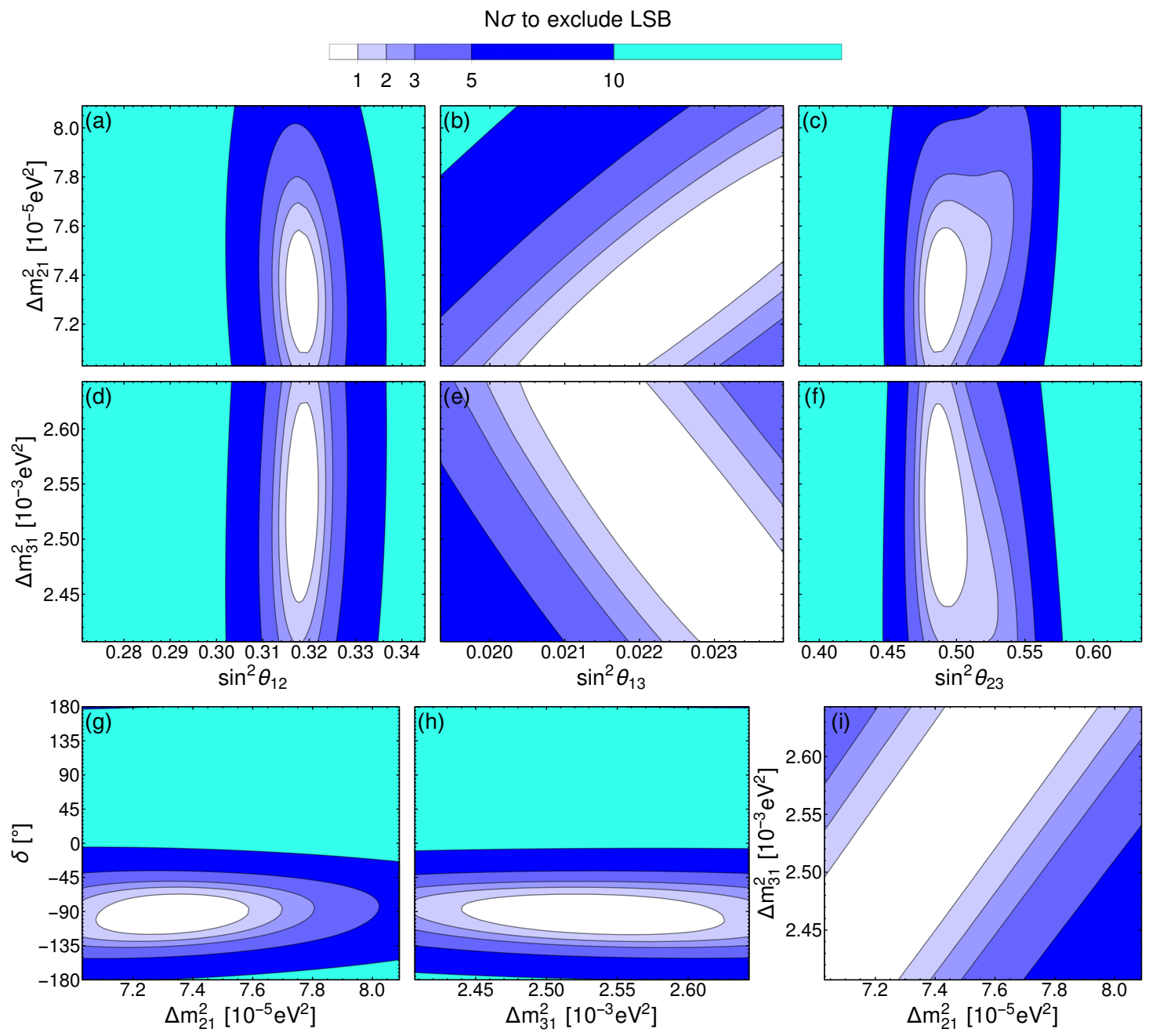

Figure 13. The predicted sensitivity of future experiments to excluding LSB, with $\eta$ fixed at $\eta=-\frac{2 \pi}{3}$, shown as a function of each pair of true parameters. The ranges of true parameters shown in the plots corresponds to the current three sigma allowed NuFIT 3.0 regions.

The ability of future experiments to exclude these models then comes from a convolution of the strength of the predictions of the model with the sensitivity of the experiments in measuring those parameters. Through our fit of the models to current global neutrino oscillation data, we have seen that the LS models make strong predictions for the values of $\theta_{12}, \theta_{23}$, and $\delta$, the three parameters for which current measurements are weakest. In addition we find that, for certain combinations of the remaining observables, $\theta_{13}, \Delta m_{21}^{2}$ and $\Delta m_{31}^{2}$, the LS models predict strong correlations.

With future experiments expected to improve precision on all six parameters measured through oscillations, our simulations have shown that the LS models can be thoroughly tested through future precise individual measurements of $\theta_{12}, \theta_{23}$, and $\delta$. This can be readily understood since the free parameters of the LS models are currently most constrained by the precise measurements of $\theta_{13}, \Delta m_{21}^{2}$ and $\Delta m_{31}^{2}$, leading to predictions for the currently less well determined parameters $\theta_{12}, \theta_{23}$, and $\delta$. 
The predictivity of the LS models means that an even higher precision measurement of those parameters which currently drive the fit of the input parameters, namely $\theta_{13}, \Delta m_{21}^{2}$ and $\Delta m_{31}^{2}$, could still exclude the LS models when considered in combination with each other. For example, the combination of any two of them could require a region of LS parameter space already excluded by the third.

These above results all highlight the strong complementarity between different classes of oscillation experiment. While the long baseline accelerator experiments DUNE and T2HK are expected to provide the strongest measurements of $\theta_{23}$ and $\delta$ (two of those that can individually test the model's viability) the third, $\theta_{12}$, will come from medium baseline reactor experiments such as JUNO and RENO-50. The strongest complementarity, however, comes from combining precision measurements of $\Delta m_{21}^{2}, \Delta m_{31}^{2}$ and $\theta_{13}$, where any pair of these measurements relies on the results from all the different experiments: long-baseline accelerator experiments for $\Delta m_{31}^{2}$, medium-baseline reactor experiments for $\Delta m_{21}^{2}$, and short-baseline reactor experiments for $\theta_{13}$.

In summary, the work presented in this paper shows that the most straightforward way to exclude the LS model is to provide a better individual determination of the three currently less precisely measured parameters $\theta_{12}, \theta_{23}$, and $\delta$, which requires both medium baseline experiments such as JUNO and RENO-50, and long baseline experiments such as DUNE and T2HK, where the synergy between the latter two experiments is thoroughly explored in [70]. In addition, the LS model could be constrained by combined measurements of the three remaining parameters $\Delta m_{21}^{2}, \Delta m_{31}^{2}$ and $\theta_{13}$, where an even higher precision of the latter reactor parameter at the short baseline Daya Bay experiment can also play an important role.

We remark that, although the above conclusions have been established for the LSA and LSB models, similar arguments can be expected to apply to any highly predictive flavour models which determine the oscillation parameters from a smaller number of input model parameters. In any such model, the input parameters will tend to be tuned to fit the strong constraints from the most precisely measured parameters, leading to predictions of the other parameters. If the models can accommodate individual measurements in this way, distinguishing between them using those parameters which drive the fit is still possible, if those models are highly constrained, but this requires the parameter measurements to be considered in combination.

In conclusion, the need for future reactor and accelerator experiments to measure individually $\theta_{12}, \theta_{23}$ and $\delta$, plus combinations of $\theta_{13}, \Delta m_{21}^{2}$ and $\Delta m_{31}^{2}$, may be considered to be general requirements in order to probe predictive flavour symmetry models. Therefore a broad programme of such precision experiments seems to be essential in order to take the next step in understanding neutrino oscillations in the context of the flavour puzzle of the Standard Model.

\section{Acknowledgments}

We would like to thank Michel Sorel, Alan Bross and Ao Liu for providing experimental information for use in our simulation of DUNE, and also the Hyper-Kamiokande protocollaboration collaboration for information used in our simulations for Hyper-Kamiokande. 
PB, SP and TC acknowledge partial support from the European Research Council under ERC Grant "NuMass" (FP7-IDEAS-ERC ERC-CG 617143). We all acknowledge partial support from ELUSIVES ITN (H2020-MSCA-ITN-2015, GA-2015674896-ELUSIVES), and InvisiblesPlus RISE (H2020-MSCARISE-2015, GA-2015-690575InvisiblesPlus). SP gratefully acknowledges partial support from the Wolfson Foundation and the Royal Society.

\section{A Exact expressions for LS sum rules}

The following expressions represent relations between the neutrino mixing parameters, newly derived and presented here for the first time. The angles and Dirac phase can be written as

$$
\sin ^{2} \theta_{13}=s(r), \quad \tan ^{2} \theta_{12}=t(r), \quad \cos 2 \theta_{23}= \pm c(r), \quad \cos \delta= \pm d(r),
$$

with positive signs taken for LSA and negative for LSB and where

$$
\begin{aligned}
& s(r)=\frac{1}{6}\left(1-\frac{55 r^{2}+4(1-4 r)}{\sqrt{\left((11 r)^{2}+4(1-7 r)\right)\left((11 r)^{2}+4(1-r)\right)}}\right) \\
& t(r)=\frac{1}{4}\left(1+\frac{55 r^{2}+4(1-4 r)}{\sqrt{\left((11 r)^{2}+4(1-7 r)\right)\left((11 r)^{2}+4(1-r)\right)}}\right) \\
& c(r)=\frac{2 r(11 r-1)\left(55 r^{2}-16 r+4-5 \sqrt{\left((11 r)^{2}+4(1-7 r)\right)\left((11 r)^{2}+4(1-r)\right)}\right)}{\left((11 r)^{2}+4(1-7 r)\right)\left((11 r)^{2}+4(1-r)\right)+4 r^{2}\left((11 r)^{2}+2(2-11 r)\right)} \\
& d(r)=-\frac{c(r)(1-5 s(r))}{2 \sqrt{2 s(r)\left(1-c(r)^{2}\right)(1-3 s(r))}} .
\end{aligned}
$$

Similar expressions for the Majorana phases also possible. Combining these, expressions relating any two of the angles and/or phases can be found. The first such relation, relating $\theta_{13}$ and $\theta_{12}$, is the same as eq. (2.6), which is general for all $\operatorname{CSD}(n)$. New exact relations between $\theta_{13}$ and $\theta_{23}$ or $\theta_{12}$ and $\theta_{23}$, as well as the relation between $\delta$ and $\theta_{12}$, true for LSA with $\eta=\frac{2 \pi}{3}$ or LSB with $\eta=-\frac{2 \pi}{3}$, are found of the form

$$
f_{ \pm}\left(\theta_{13}, \theta_{23}\right)=0, \quad g_{ \pm}\left(\theta_{12}, \theta_{23}\right)=0, \quad h_{ \pm}\left(\delta, \theta_{12}\right)=0,
$$

where again the positive (negative) sign is used in the functions valid for LSA (LSB). Exact expressions are given as

$$
\begin{aligned}
& f_{ \pm}\left(\theta_{13}, \theta_{23}\right)=\frac{44 s_{13}^{2} \sqrt{1-3 s_{13}^{2}}}{4\left(1-6 s_{13}^{2}\right) \mp 3 c_{13}^{2} \cos 2 \theta_{23}} \pm \frac{c_{13}^{2} \cos 2 \theta_{23}}{\sqrt{1-3 s_{13}^{2}}}-\sqrt{\frac{8 s_{13}^{2}}{3}-\frac{c_{13}^{4} \cos ^{2} 2 \theta_{23}}{3\left(1-3 s_{13}^{2}\right)}}, \\
& g_{ \pm}\left(\theta_{12}, \theta_{23}\right)=\frac{22 s_{12}^{2} \sqrt{1-3 s_{12}^{2}}}{2\left(5 s_{12}^{2}-1\right) \mp \cos 2 \theta_{23}} \pm \frac{\cos 2 \theta_{23}}{\sqrt{1-3 s_{12}^{2}}}-\sqrt{4 s_{12}^{2}-\frac{\cos ^{2} 2 \theta_{23}}{3\left(1-3 s_{12}^{2}\right)}}, \\
& h_{ \pm}\left(\delta, \theta_{12}\right)=\frac{5 s_{12}^{2}-1}{s_{12} \sqrt{1-3 s_{12}^{2}}} \pm \frac{\sqrt{3} \cos \delta}{\sqrt{1-12 s_{12}^{2}\left(1-3 s_{12}^{2}\right) \sin ^{2} \delta}}+\frac{11 \sqrt{1-12 s_{12}^{2}\left(1-3 s_{12}^{2}\right) \sin ^{2} \delta}}{2\left(6 s_{12}^{2}-1\right) \sin \delta \mp 2 \sqrt{3} \cos \delta}
\end{aligned}
$$


Open Access. This article is distributed under the terms of the Creative Commons Attribution License (CC-BY 4.0), which permits any use, distribution and reproduction in any medium, provided the original author(s) and source are credited.

\section{References}

[1] T. Ohlsson ed., Special Issue on "Neutrino Oscillations: Celebrating the Nobel Prize in Physics 2015", Nucl. Phys. B 908 (2016) 1.

[2] S.F. King and C. Luhn, Neutrino Mass and Mixing with Discrete Symmetry, Rept. Prog. Phys. 76 (2013) 056201 [arXiv: 1301.1340] [INSPIRE].

[3] S.F. King, Neutrino mass models, Rept. Prog. Phys. 67 (2004) 107 [hep-ph/0310204] [INSPIRE].

[4] H. Ishimori, T. Kobayashi, H. Ohki, Y. Shimizu, H. Okada and M. Tanimoto, Non-Abelian Discrete Symmetries in Particle Physics, Prog. Theor. Phys. Suppl. 183 (2010) 1 [arXiv: 1003.3552] [INSPIRE].

[5] S.F. King, A. Merle, S. Morisi, Y. Shimizu and M. Tanimoto, Neutrino Mass and Mixing: from Theory to Experiment, New J. Phys. 16 (2014) 045018 [arXiv:1402.4271] [InSPIRE].

[6] S.F. King, Models of Neutrino Mass, Mixing and CP-violation, J. Phys. G 42 (2015) 123001 [arXiv: 1510.02091] [INSPIRE].

[7] P. Minkowski, $\mu \rightarrow e \gamma$ at a Rate of One Out of $10^{9}$ Muon Decays?, Phys. Lett. B 67 (1977) 421 [INSPIRE].

[8] M. Gell-Mann, P. Ramond and R. Slansky, Complex Spinors and Unified Theories, Conf. Proc. C 790927 (1979) 315 [arXiv:1306.4669] [INSPIRE].

[9] T. Yanagida, Horizontal Symmetry And Masses Of Neutrinos, Conf. Proc. C 7902131 (1979) 95 [INSPIRE].

[10] S.L. Glashow, The Future of Elementary Particle Physics, in proceedings of Cargese Summer Institute: Quarks and Leptons, Cargese, France, 9-29 July 1979 [NATO Sci. Ser. B 61 (1980) 687] [INSPIRE].

[11] R.N. Mohapatra and G. Senjanović, Neutrino Mass and Spontaneous Parity Violation, Phys. Rev. Lett. 44 (1980) 912 [inSPIRE].

[12] J. Schechter and J.W.F. Valle, Neutrino Masses in $\mathrm{SU}(2) \times \mathrm{U}(1)$ Theories, Phys. Rev. D 22 (1980) 2227 [INSPIRE].

[13] S.F. King, Neutrino Mass and Mixing in the Seesaw Playground, Nucl. Phys. B 908 (2016) 456 [arXiv: 1511.03831] [INSPIRE].

[14] S.F. King, Large mixing angle MSW and atmospheric neutrinos from single right-handed neutrino dominance and U(1) family symmetry, Nucl. Phys. B 576 (2000) 85 [hep-ph/9912492] [INSPIRE].

[15] S.F. King, Constructing the large mixing angle MNS matrix in seesaw models with right-handed neutrino dominance, JHEP 09 (2002) 011 [hep-ph/0204360] [INSPIRE].

[16] P.H. Frampton, S.L. Glashow and T. Yanagida, Cosmological sign of neutrino CP-violation, Phys. Lett. B 548 (2002) 119 [hep-ph/0208157] [INSPIRE].

[17] K. Harigaya, M. Ibe and T.T. Yanagida, Seesaw Mechanism with Occam's Razor, Phys. Rev. D 86 (2012) 013002 [arXiv:1205.2198] [INSPIRE]. 
[18] S.F. King, Minimal predictive see-saw model with normal neutrino mass hierarchy, JHEP 07 (2013) 137 [arXiv: 1304.6264] [INSPIRE].

[19] F. Björkeroth, F.J. de Anda, I. de Medeiros Varzielas and S.F. King, Towards a complete $A_{4} \times \mathrm{SU}(5)$ SUSY GUT, JHEP 06 (2015) 141 [arXiv:1503.03306] [inSPIRE].

[20] F. Björkeroth, F.J. de Anda, I. de Medeiros Varzielas and S.F. King, Towards a complete $\Delta(27) \times \mathrm{SO}(10)$ SUSY GUT, Phys. Rev. D 94 (2016) 016006 [arXiv:1512.00850] [INSPIRE].

[21] F. Björkeroth, F.J. de Anda, I. de Medeiros Varzielas and S.F. King, Leptogenesis in minimal predictive seesaw models, JHEP 10 (2015) 104 [arXiv:1505.05504] [INSPIRE].

[22] S.F. King, Atmospheric and solar neutrinos with a heavy singlet, Phys. Lett. B 439 (1998) 350 [hep-ph/9806440] [inSPIRE].

[23] S.F. King, Atmospheric and solar neutrinos from single right-handed neutrino dominance and U(1) family symmetry, Nucl. Phys. B 562 (1999) 57 [hep-ph/9904210] [INSPIRE].

[24] S.F. King, Predicting neutrino parameters from $\mathrm{SO}(3)$ family symmetry and quark-lepton unification, JHEP 08 (2005) 105 [hep-ph/0506297] [INSPIRE].

[25] S. Antusch, S.F. King, C. Luhn and M. Spinrath, Trimaximal mixing with predicted $\theta_{13}$ from a new type of constrained sequential dominance, Nucl. Phys. B 856 (2012) 328 [arXiv: 1108.4278] [INSPIRE].

[26] S.F. King, Minimal see-saw model predicting best fit lepton mixing angles, Phys. Lett. B 724 (2013) 92 [arXiv: 1305.4846] [INSPIRE].

[27] S.F. King, A model of quark and lepton mixing, JHEP 01 (2014) 119 [arXiv:1311.3295] [INSPIRE].

[28] S.F. King, A to Z of Flavour with Pati-Salam, JHEP 08 (2014) 130 [arXiv:1406.7005] [INSPIRE].

[29] F. Björkeroth and S.F. King, Testing constrained sequential dominance models of neutrinos, J. Phys. G 42 (2015) 125002 [arXiv:1412.6996] [INSPIRE].

[30] S.F. King, Littlest Seesaw, JHEP 02 (2016) 085 [arXiv: 1512.07531] [INSPIRE].

[31] S.F. King and C. Luhn, Littlest Seesaw model from $S_{4} \times$ U(1), JHEP 09 (2016) 023 [arXiv: 1607.05276] [INSPIRE].

[32] S.F. King and C. Luhn, On the origin of neutrino flavour symmetry, JHEP 10 (2009) 093 [arXiv:0908.1897] [INSPIRE].

[33] Z.-z. Xing and S. Zhou, Tri-bimaximal Neutrino Mixing and Flavor-dependent Resonant Leptogenesis, Phys. Lett. B 653 (2007) 278 [hep-ph/0607302] [INSPIRE].

[34] C.H. Albright, A. Dueck and W. Rodejohann, Possible Alternatives to Tri-bimaximal Mixing, Eur. Phys. J. C 70 (2010) 1099 [arXiv: 1004.2798] [InSPIRE].

[35] X.-G. He and A. Zee, Minimal Modification to Tri-bimaximal Mixing, Phys. Rev. D 84 (2011) 053004 [arXiv: 1106.4359] [INSPIRE].

[36] W. Rodejohann and H. Zhang, Simple two Parameter Description of Lepton Mixing, Phys. Rev. D 86 (2012) 093008 [arXiv:1207.1225] [INSPIRE].

[37] I. de Medeiros Varzielas and L. Lavoura, Flavour models for TM $M_{1}$ lepton mixing, J. Phys. G 40 (2013) 085002 [arXiv:1212.3247] [INSPIRE].

[38] W. Grimus, Discrete symmetries, roots of unity and lepton mixing, J. Phys. G 40 (2013) 075008 [arXiv: 1301.0495 ] [INSPIRE]. 
[39] C.H. Albright and W. Rodejohann, Comparing Trimaximal Mixing and Its Variants with Deviations from Tri-bimaximal Mixing, Eur. Phys. J. C 62 (2009) 599 [arXiv:0812.0436] [INSPIRE].

[40] P.F. Harrison, D.H. Perkins and W.G. Scott, Tri-bimaximal mixing and the neutrino oscillation data, Phys. Lett. B 530 (2002) 167 [hep-ph/0202074] [INSPIRE].

[41] C. Luhn, Trimaximal TM $M_{1}$ neutrino mixing in $S_{4}$ with spontaneous CP-violation, Nucl. Phys. B 875 (2013) 80 [arXiv:1306.2358] [INSPIRE].

[42] G.-J. Ding, S.F. King, C. Luhn and A.J. Stuart, Spontaneous CP-violation from vacuum alignment in $S_{4}$ models of leptons, JHEP 05 (2013) 084 [arXiv:1303.6180] [INSPIRE].

[43] F. Feruglio, C. Hagedorn and R. Ziegler, A realistic pattern of lepton mixing and masses from $S_{4}$ and CP, Eur. Phys. J. C 74 (2014) 2753 [arXiv:1303.7178] [InSPIRE].

[44] S. Antusch, S.F. King, C. Luhn and M. Spinrath, Right Unitarity Triangles and Tri-Bimaximal Mixing from Discrete Symmetries and Unification, Nucl. Phys. B 850 (2011) 477 [arXiv: 1103.5930] [INSPIRE].

[45] S. Boudjemaa and S.F. King, Deviations from Tri-bimaximal Mixing: Charged Lepton Corrections and Renormalization Group Running, Phys. Rev. D 79 (2009) 033001 [arXiv:0808.2782] [INSPIRE].

[46] I. Esteban, M.C. Gonzalez-Garcia, M. Maltoni, I. Martinez-Soler and T. Schwetz, Updated fit to three neutrino mixing: exploring the accelerator-reactor complementarity, JHEP 01 (2017) 087 [arXiv:1611.01514] [INSPIRE].

[47] K. Iwamoto, Recent Results from T2K and Future Prospects, talk given at The 38th International Conference on High Energy Physics, Chicago, U.S.A., 3-10 August 2016.

[48] P. Vahle, New results from NOvA, talk given at The XXVII International Conference on Neutrino Physics and Astrophysics, London, U.K., 4-9 July 2016.

[49] F. Capozzi, G.L. Fogli, E. Lisi, A. Marrone, D. Montanino and A. Palazzo, Status of three-neutrino oscillation parameters, circa 2013, Phys. Rev. D 89 (2014) 093018 [arXiv: 1312.2878] [INSPIRE].

[50] D.V. Forero, M. Tortola and J.W.F. Valle, Neutrino oscillations refitted, Phys. Rev. D 90 (2014) 093006 [arXiv:1405.7540] [INSPIRE].

[51] F. Borzumati and A. Masiero, Large Muon and electron Number Violations in Supergravity Theories, Phys. Rev. Lett. 57 (1986) 961 [InSPIRE].

[52] J. Hisano, T. Moroi, K. Tobe and M. Yamaguchi, Lepton flavor violation via right-handed neutrino Yukawa couplings in supersymmetric standard model, Phys. Rev. D 53 (1996) 2442 [hep-ph/9510309] [INSPIRE].

[53] S.F. King and M. Oliveira, Lepton flavor violation in string inspired models, Phys. Rev. D 60 (1999) 035003 [hep-ph/9804283] [INSPIRE].

[54] M. Dimou, S.F. King and C. Luhn, Approaching Minimal Flavour Violation from an $\mathrm{SU}(5) \times S_{4} \times \mathrm{U}(1) S U S Y$ GUT, JHEP 02 (2016) 118 [arXiv:1511.07886] [INSPIRE].

[55] M. Dimou, S.F. King and C. Luhn, Phenomenological implications of an $\mathrm{SU}(5) \times S_{4} \times \mathrm{U}(1)$ SUSY GUT of flavor, Phys. Rev. D 93 (2016) 075026 [arXiv:1512.09063] [INSPIRE].

[56] T. Blazek and S.F. King, Lepton flavor violation in the constrained MSSM with natural neutrino mass hierarchy, Nucl. Phys. B 662 (2003) 359 [hep-ph/0211368] [INSPIRE].

[57] S.F. King and C. Luhn, Trimaximal neutrino mixing from vacuum alignment in A4 and $\mathrm{S}_{4}$ models, JHEP 09 (2011) 042 [arXiv:1107.5332] [INSPIRE]. 
[58] P. Ballett, S.F. King, C. Luhn, S. Pascoli and M.A. Schmidt, Testing atmospheric mixing sum rules at precision neutrino facilities, Phys. Rev. D 89 (2014) 016016 [arXiv:1308.4314] [INSPIRE].

[59] P. Ballett, S.F. King, C. Luhn, S. Pascoli and M.A. Schmidt, Precision measurements of $\theta_{12}$ for testing models of discrete leptonic flavour symmetries, J. Phys. Conf. Ser. 598 (2015) 012014 [arXiv: 1406.0308] [INSPIRE].

[60] C. Jarlskog, Commutator of the Quark Mass Matrices in the Standard Electroweak Model and a Measure of Maximal CP-violation, Phys. Rev. Lett. 55 (1985) 1039 [InSPIRE].

[61] Hyper-KamioKande proto-collaboration, K. Abe et al., Hyper-Kamiokande Design Report, KEK Preprint 2016-21, ICRR-Report-701-2016-1 (2016) [https://lib-extopc.kek.jp/preprints/PDF/2016/1627/1627021.pdf] [INSPIRE].

[62] Hyper-Kamiokande proto-collaboration, K. Abe et al., Physics potential of a long-baseline neutrino oscillation experiment using a J-PARC neutrino beam and Hyper-Kamiokande, PTEP 2015 (2015) $053 \mathrm{C} 02$ [arXiv:1502.05199] [INSPIRE].

[63] DUNE collaboration, R. Acciarri et al., Long-Baseline Neutrino Facility (LBNF) and Deep Underground Neutrino Experiment (DUNE). Volume 1: The LBNF and DUNE Projects, arXiv: 1601.05471 [INSPIRE].

[64] DUNE collaboration, R. Acciarri et al., Long-Baseline Neutrino Facility (LBNF) and Deep Underground Neutrino Experiment (DUNE). Volume 2: The Physics Program for DUNE at $L B N F$, arXiv:1512.06148 [INSPIRE].

[65] DAYA BAY collaboration, X. Guo et al., A precision measurement of the neutrino mixing angle $\theta_{13}$ using reactor antineutrinos at Daya-Bay, hep-ex/0701029 [INSPIRE].

[66] J. Cao and K.-B. Luk, An overview of the Daya Bay Reactor Neutrino Experiment, Nucl. Phys. B 908 (2016) 62 [arXiv: 1605.01502] [InSPIRE].

[67] JUNO collaboration, Z. Djurcic et al., JUNO Conceptual Design Report, arXiv:1508.07166 [INSPIRE].

[68] P. Huber, M. Lindner and W. Winter, Simulation of long-baseline neutrino oscillation experiments with GLoBES (General Long Baseline Experiment Simulator), Comput. Phys. Commun. 167 (2005) 195 [hep-ph/0407333] [INSPIRE].

[69] P. Huber, J. Kopp, M. Lindner, M. Rolinec and W. Winter, New features in the simulation of neutrino oscillation experiments with GLoBES 3.0: General Long Baseline Experiment Simulator, Comput. Phys. Commun. 177 (2007) 432 [hep-ph/0701187] [INSPIRE].

[70] P. Ballett, S.F. King, S. Pascoli, N.W. Prouse and T. Wang, Sensitivities and synergies of DUNE and T2HK, arXiv:1612.07275 [INSPIRE].

[71] S.-B. Kim, New results from RENO and prospects with RENO-50, Nucl. Part. Phys. Proc. 265-266 (2015) 93 [arXiv:1412.2199] [INSPIRE].

[72] F. Ardellier et al., Letter of intent for Double-CHOOZ: A search for the mixing angle $\theta_{13}$, hep-ex/0405032 [INSPIRE].

[73] RENO collaboration, J.K. Ahn et al., RENO: An Experiment for Neutrino Oscillation Parameter $\theta_{13}$ Using Reactor Neutrinos at Yonggwang, arXiv:1003.1391 [INSPIRE].

[74] S.S. Wilks, The Large-Sample Distribution of the Likelihood Ratio for Testing Composite Hypotheses, Ann. Math. Statist. 9 (1938) 60. 